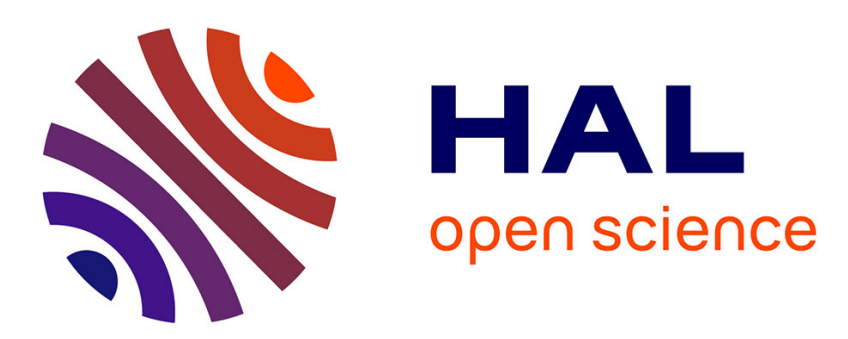

\title{
The Asymptotic Behaviour of the Fourier Transforms of Orthogonal Polynomials I: Mellin Transform Techniques
}

Sandro Vaienti, Giorgio Mantica

\section{To cite this version:}

Sandro Vaienti, Giorgio Mantica. The Asymptotic Behaviour of the Fourier Transforms of Orthogonal Polynomials I: Mellin Transform Techniques. Annales Henri Poincaré, 2007, 8 (2), pp.265-300. 10.1007/s00023-006-0308-2 . hal-00476268

\section{HAL Id: hal-00476268 \\ https://hal.science/hal-00476268}

Submitted on 25 Apr 2010

HAL is a multi-disciplinary open access archive for the deposit and dissemination of scientific research documents, whether they are published or not. The documents may come from teaching and research institutions in France or abroad, or from public or private research centers.
L'archive ouverte pluridisciplinaire HAL, est destinée au dépôt et à la diffusion de documents scientifiques de niveau recherche, publiés ou non, émanant des établissements d'enseignement et de recherche français ou étrangers, des laboratoires publics ou privés. 


\title{
The Asymptotic Behaviour of the Fourier Transforms of Orthogonal Polynomials I: Mellin Transform Techniques
}

\author{
Giorgio Mantica and Sandro Vaienti
}

\begin{abstract}
The Fourier transform of orthogonal polynomials with respect to their own orthogonality measure defines the family of Fourier-Bessel functions. We study the asymptotic behaviour of these functions and of their products, for large real values of the argument. By employing a Mellin analysis we construct a general framework to exhibit the relation of the asymptotic decay laws to certain dimensions of the orthogonality measure, that are defined via the divergence abscissas of suitable integrals. The unifying rôle of Mellin transform techniques in deriving classical and new results is underlined.
\end{abstract}

2000 Math. Subj. Class: 42C05, 33E20, 28A80, 30E15, 30E20

Keywords: singular measures, Fourier transform, dimensions, orthogonal polynomials

\section{Introduction}

This is the first of two papers on the asymptotic behaviour of the Fourier transforms of the orthogonal polynomials of a measure. Let $\mu$ be a positive Borel measure supported on the real axis that we suppose to be normalized, and for which the moment problem is determined [1]: that is, all moments $\mu_{n}:=\int d \mu(s) s^{n}$, $n \in \mathbb{N}$ exist, and uniquely identify the measure. This class includes measures with unbounded support, as well as compactly supported multi-fractal measures, that are the principal motivation for this study. We then consider the set of associated orthogonal polynomials $\left\{p_{n}(\mu ; s)\right\}_{n \in \mathbb{N}}$,

$$
\int d \mu(s) p_{n}(\mu ; s) p_{m}(\mu ; s)=\delta_{n m}
$$

where $\delta_{n m}$ is the Kronecker delta. We adopt the convention that $\int d \mu(s)$ means applying the integration with respect to the measure $\mu$ to all functions of the variable $s$ that follow to the right. Termination of the integral will be imposed, when not self-evident, by closing it into parentheses. For convenience and consistency, 
the same ordering of symbols will also be used for Riemann integrals. Let us now define:

Definition 1 The generalized Fourier-Bessel Functions (F-B. functions for short) $\mathcal{J}_{n}(\mu ; t)$ are the Fourier transforms of the orthogonal polynomials $p_{n}(\mu ; s)$ with respect to $\mu$ :

$$
\mathcal{J}_{n}(\mu ; t):=\int d \mu(s) p_{n}(\mu ; s) e^{-i t s} .
$$

This paper is concerned with the investigation of the long-time behaviour of the temporal Cesaro averages of the generalized F-B. functions,

$$
\overline{\mathcal{J}}_{n}(\mu ; t):=\mathcal{C}\left(\mathcal{J}_{n} ; t\right):=\frac{1}{2 t} \int_{-t}^{t} d t^{\prime} \mathcal{J}_{n}\left(\mu ; t^{\prime}\right) .
$$

Here and in the following $\mathcal{C}(f ; t)$ and $\bar{f}$ indicate the symmetric Cesaro average of a function $f$. For F-B. functions this can be rewritten as

$$
\overline{\mathcal{J}}_{n}(\mu ; t)=\int d \mu(s) \Phi(t, s) p_{n}(\mu ; s),
$$

where $\Phi(t, s)=\sin (t s) / t s=\operatorname{sinc}(t s)$. Eq. (4) also makes it evident that $\overline{\mathcal{J}}_{n}(\mu ; t)$ is a real quantity. Comparison with eq. (2) shows that the same notation can be employed for instantaneous values, letting $\Phi(t, s)=e^{-i t s}$. The techniques presented here can treat this case equally well, although with different results when $\mu$ is singular continuous. In fact, recourse to Cesaro averaging is dictated in this case by the presence of intermittent oscillations of the $F$ - $B$. functions [2].

We shall also study the Cesaro averages of products of two $F$ - $B$. functions:

Definition 2 The quadratic amplitudes $A_{n m}(\mu ; t)$ are defined as:

$$
A_{n m}(\mu ; t):=\mathcal{C}\left(\mathcal{J}_{n} \mathcal{J}_{m}^{*} ; t\right):=\frac{1}{2 t} \int_{-t}^{t} d t^{\prime} \mathcal{J}_{n}\left(\mu ; t^{\prime}\right) \mathcal{J}_{m}^{*}\left(\mu ; t^{\prime}\right)
$$

where the star indicates complex conjugation. This problem, for the case $n=m=$ 0 is classical in the literature, and a variety of methods for its solution have been proposed, a brief review of which is outlined in the next section.

In this paper, we will discuss a class of techniques based on the Mellin transform (M.T. for short) that permit to recover the existing results into a global theory, and to obtain new ones. We adopt the following definition of the Mellin transform of a function $f$ :

$$
\mathcal{M}(f ; z):=\int_{0}^{\infty} d t f(t) t^{z-1} .
$$

The precise meaning of this integral will be defined in the following, as convenience will demand, either as a Lebesgue integral, or as an improper Riemann integral. We 
shall consider the Mellin transforms of the Cesaro averages $\overline{\mathcal{J}}_{n}(\mu ; t)$ and $A_{n m}(\mu ; t)$, that we shall call $M_{n}(\mu ; z)$ and $M_{n m}(\mu ; z)$, respectively, letting the number of subscripts discriminate between the two cases.

Let now $f$ be positive: in our case, the diagonal amplitudes $\mathcal{J}_{n} \mathcal{J}_{n}^{*}$ are such. Then, it is well known that $\mathcal{M}(f ; z)$ is analytic in a strip $\zeta_{0}<\Re(z)<\zeta_{\infty}$ in the complex plane. This domain of analyticity of the Mellin transform is indicative of the short and long-time behaviour of $f(t)$. Since we are mainly interested in the latter, we shall investigate the right divergence abscissa of the Mellin transform, $\zeta_{\infty}$. It is an easy exercise to show that $\zeta_{\infty}$ interpolates the upper and lower limits of $-\log f(t) / \log (t)$, for $t \rightarrow \infty$. Indeed, in a number of cases, equality with the upper limit follows as a consequence of a classical theorem. It will be apparent in the following that positivity of the function $f$ is a strong constraint in this problem. Nonetheless, we shall find that useful results can be obtained via the M.T. technique also in the case of the non necessarily positive functions $\overline{\mathcal{J}}_{n}(\mu ; t)$ and $A_{n m}(\mu ; t)$, with $n \neq m$.

In this analysis, a key rôle will be played by potential theoretic quantities. First, we shall consider

Definition 3 The generalized electrostatic potential $\mathcal{G}(\mu ; s, z)$ of the measure $\mu$ at the point $s \in \mathbb{C}$ is

$$
\mathcal{G}(\mu ; s, z):=\int d \mu(r) \frac{1}{|r-s|^{z}} .
$$

Integrating the potential with respect to $\mu$ leads to:

Definition 4 The generalized electrostatic energy $\mathcal{E}(\mu ; z)$ of the measure $\mu$ is:

$$
\mathcal{E}(\mu ; z):=\iint d \mu(r) d \mu(s) \frac{1}{|r-s|^{z}} .
$$

It is immediate to see that eqs. (7) and (8) define analytic functions in a half plane. Their divergence abscissas can be used to define two important quantities:

Definition 5 The electrostatic local dimension $d(\mu ; s)$ of a measure $\mu$ at the point $s$ is the divergence abscissa of the generalized electrostatic potential $\mathcal{G}(\mu ; s, z)$ :

$$
d(\mu ; s):=\sup \{x \in \mathbb{R} \text { s.t. } \mathcal{G}(\mu ; s, x)<\infty\} .
$$

Definition 6 The electrostatic correlation dimension $D_{2}(\mu)$ of a measure $\mu$ is the divergence abscissa of the generalized electrostatic energy $\mathcal{E}(\mu ; z)$ :

$$
D_{2}(\mu):=\sup \{x \in \mathbb{R} \text { s.t. } \mathcal{E}(\mu ; x)<\infty\} .
$$


We shall prove that these dimensions are related by

Theorem 1 The electrostatic local dimension of $\mu$ at any point $s, d(\mu ; s)$, is larger than, or equal to, one-half of the electrostatic correlation dimension of the measure $\mu, D_{2}(\mu)$ :

$$
d(\mu ; s) \geq \frac{1}{2} D_{2}(\mu) \forall s \in \mathbb{R} .
$$

We shall prove this theorem, together with an almost-sure variant in Sect. 16.

The electrostatic dimensions coincide with the corresponding lower dimensions of conventional theory, that we define below, as proven in [3], [4]. Indeed, these results follow from a quite general theorem on the Mellin transform of Stieltjes measures that is implicit in the literature, and that we shall spell out and prove in the following.

We will show in this paper that the electrostatic dimensions are related to the right divergence abscissas of $M_{n}(\mu ; z)$ and $M_{n m}(\mu ; z)$ : therefore, they control the asymptotic behaviour of $\overline{\mathcal{J}}_{n}(\mu ; t)$ and $A_{n m}(\mu ; t)$ for large $t$, for any measure $\mu$. Proper analytical tools will be developed and utilized to give a precise meaning to the asymptotic relation. In addition, relations between the asymptotic behaviours of the two quantities will be brought to light. In this process, side results of some interest will be developed, involving, among other things, the asymptotic behaviour of the potential $\mathcal{G}(\mu ; s, z)$ when $z$ tends to infinity in the analyticity strip in the complex plane.

The organization of this paper is the following: in the next section we shall attempt a critical discussion of the results that have already appeared in the literature on particular subcases of our problem, or that have dealt with a restricted set of situations: we hope to convince the reader that the Mellin approach achieves the widest generality. In section 3 we prove a theorem that contains those in [3], [4] mentioned above as particular cases. A variant of this theorem is applied in Sect. 4 to re-derive a classical result on the decay of the Fourier transform of a measure.

We then start the analysis of the Mellin transform of the averaged F-B. functions. In Sect. 5 we introduce asymptotic exponents defined "electrostatically" that control their behaviour at infinity via a general decay theorem. Variants of this theorem will be proven in the successive sections via different techniques, based on the inversion of the Mellin transform. In Sect. 6 we put in relation the local properties of the measure $\mu$ at zero and the analyticity structure of the Mellin transform of F-B. functions. This information is then utilized in Sect. 7 to set up an inverse transform technique. This leads to the proof of a power-law bound on the asymptotic decay of the averaged F-B. functions, in a restricted range of exponents. In Sect. 8 the inversion theory, in a weak form originally due to Makarov [5], is applied in a larger set of exponents. Next, in Sect. 9, an elementary application of integration by parts in a Riemann integral, combined with a computation of the Mellin transform of non-averaged F.B. functions, permits to obtain the asymptotic 
decay in strong form over a larger set of exponents, so to overcome the limitations of the previous two sections.

Starting from Sect. 10 we turn our attention to the quadratic amplitudes $A_{n m}(\mu ; t)$, much in line with the methodology of the preceding sections. We first put in relation the analyticity structure of their Mellin transform with the dimensional properties of the measure $\mu$, and we prove a general decay theorem. These properties are then utilised in Sect. 11 to set up the Mellin inversion procedure. Positivity properties of the diagonal quadratic amplitudes (that is, the amplitudes of physical significance in quantum mechanics) are employed in Sect. 12 to prove the asymptotic decay of in strong form, for the widest possible set of exponents. The same strong result is obtained in Sect. 13 for the non-diagonal amplitudes, still following the techniques developed for linear quantities.

A new object is analyzed in Sect. 14: the asymptotic behaviour of the Mellin transform of the F-B. functions when the argument goes to infinity in the vertical strip of analyticity. We show that the techniques of the previous sections can be applied to these quantities, by introducing a class of measures, $\nu_{x}(\mu)$, constructed upon the original measure $\mu$, and the abscissa $x$ in the analyticity strip. Under the restrictive hypothesis of boundedness of the support of $\mu$, the relations between $D_{2}(\mu)$ and $D_{2}\left(\nu_{x}(\mu)\right)$ are investigated. In Sect. 15 we show how this analysis can be employed to extend the $L^{1}$ property of the Mellin transform on vertical lines in the strip of analyticity.

Finally, in Sect. 16 we adapt the analysis to the local behaviour of the measure $\mu$ around any arbitrary point. We derive a lower bound on the local dimensions at all points that is equal to one half of the correlation dimension. The conclusions, Sect. 17, briefly discuss the relevance of the Mellin analysis of spectral and dynamical properties like those encountered in this paper.

A table of the mathematical symbols of frequent usage, including reference to their definitions, is added at the end of the paper. An elementary appendix exemplifies the results of this paper on a simple family of measures. In a companion paper, the theory is made explicit for the balanced invariant measure of an Iterated Function System, and the results are applied to quantum dynamics.

\section{Previous Results and the Mellin Transform Ap- proach}

It is now important to review previous results that apply to our problem. We shall not proceed in chronological order, but rather we shall move from specific to more general results. Of course, we cannot claim completeness, and we apologize in advance for unwanted omissions. First of all, since $p_{0}(\mu ; x)$ is a constant, the case $n=m=0$ leads to the Fourier transform of $\mu$. This case has obviously received a lot of attention.

The first result is Strichartz theorem [6]: it underlines the importance of the continuity properties of the measure $\mu$. A measure $\mu$ is called locally uniformly 
$\alpha$-dimensional if there exist a constant $C$ such that the measure of the ball of radius $r$ centered at $s, \mu\left(B_{r}(s)\right)$, is bounded by $C r^{\alpha}$ uniformly in $s$. In a sense, $\alpha$ is a sort of lower bound to the local dimensions of $\mu$. Under these conditions, Strichartz theorem (adapted to the $n, m$ case) predicts that

$$
\limsup _{t \rightarrow \infty} t^{\alpha} A_{n m}(\mu ; t) \leq C^{\prime},
$$

with $C^{\prime}$ another constant. A reverse inequality, this time with the liminf, can be obtained when the support of the measure is a quasi-regular set $[6,7]$. Therefore, when these conditions are met, Strichartz's analysis provides us with the leading asymptotics of the amplitudes: $A_{n m}(\mu ; t) \sim t^{-\alpha}$.

It is a matter of fact that most interesting measures are not uniformly $\alpha$ dimensional measures, or with quasi-regular support; typically, multi-fractal measures do not possess these properties. Among these, there exists a family for which the problem has been solved to a large extent, the class of Linear Iterated Functions Systems (L.I.F.S.), that we discuss in a second paper. Various techniques have been used to tackle this class, all of them relying on the self-similarity properties of the measure $[8,9,10,11,12]$. The exponent $\alpha$ of the asymptotic decay law is then determined by an implicit equation. It turns out, obviously, that this value coincides with the correlation dimension of the L.I.F.S. measure. The same result for disconnected L.I.F.S. has also been obtained following a different route, with the aid of the Mellin transform [13],[2],[5]. Our treatment starts off from the results of these references, in particular of the last, that, although devoted to the restricted case of L.I.F.S., initiated the analysis of the inversion of the Mellin transform in our problem.

The approach via the Mellin transform has the advantage of rendering the identification of the decay exponents transparent. In addition, it does not require any open set condition - except, of course, if one wants to compute explicitly such exponents. One of the goals of this paper is to show that the Mellin transform is fully general, and applies to any probability measure $\mu$, for which the moment problem is determined. Indeed, were this not the case, the results for the existing moments would still hold unchanged.

The Mellin approach is implicitly contained in some estimates on the correlation dimension in Falconer's book [14], but it was firstly employed in the present context by Bessis et al. in [13], that offers the earliest explicit proof, to our knowledge, of the relation between the asymptotic behaviour of the Fourier transform of a measure and its correlation dimension, defined as in section 1. Ref. [13] also contains the case of linear I.F.S., that was further analyzed in [15]. Since the main focus of [13] was on correlation integrals, the result may have passed unnoticed. As a matter of fact, three problems are intimately related, and lead to the generalized energy integral $\mathcal{E}(\mu ; z)$ of eq. (8): the asymptotic behavior of the Fourier transform of a measure, that of correlation integrals, and that of quantum amplitudes. In studying the last, Ketzmerick et al. [16] rediscovered the rôle of the correlation dimension by using formal manipulations that have the virtue, as it is often 
the case, of unveiling the nature of the problem. These latter were further made rigorous and extended by the use of functional analysis in [17], and by wavelet techniques in $[18,19]$, to show that $\lim \sup (\inf )_{t \rightarrow \infty} \log A_{00}(\mu ; t) / \log t$ coincide with the upper and lower correlation dimension of the measure $\mu$. In the quantum mechanical context, further results were established in [20], that we shall mention later, since they also use Mellin-like techniques. Also concerned with the relation between the continuity properties of the measure, and its Fourier transform is the work [21].

Linear quantities, leading to the local rather than the correlation dimension, have been studied in the wavelet theory [22, 23], and it the Green function analysis of measures of quantum mechanical origin [24].

In [2], [25] the Mellin technique was applied not only to F-B. functions, but also to a more cumbersome combination of these: the sums $\rho_{\gamma}(t):=\sum_{n} n^{\gamma}\left|\mathcal{J}_{n}(\mu ; t)\right|^{2}$, where $\gamma$ is a positive quantity. They can be seen as the moments of a quantum distribution over a discrete lattice, and the asymptotic behaviour of the sum of the series is different from that of its individual terms [26].

The results we present here widen the scope of these investigations in many respects: firstly, our results apply to the case of a general measure, as opposed to the specific case of L.I.F.S. measures. This specific class being of particular importance, it will be further analyzed in a companion paper. Moreover, we extend the analysis from the Fourier transform of the measure alone, to that of the related orthogonal polynomials, for all values of the index. In addition, we also investigate the non-positive quantities arising from averaging the F-B. functions themselves, a theme that has received less attention than the quadratic case. Also new is the analysis of the asymptotic behaviour of the Mellin transform itself, in the strip of analyticity, that leads to interesting speculations on a different family of measures, that we consider only briefly in this paper. Finally, we believe that collecting old and new results under the unifying theme of Mellin transform techniques is a valuable endeavour, that might bring benefit to research in different areas.

\section{$3 \quad$ Electrostatic and generalized dimensions}

The relation of the electrostatic dimensions defined in Sect. 1, and the more conventional generalized dimensions is well known. It can be seen as a particular case of a general theorem on Mellin (and Laplace) transforms that is presented in this section. Let us first recall what the conventional quantities are:

Definition 7 The upper (lower) local dimensions $\gamma_{ \pm}(\mu ; s)$ of a measure $\mu$ at the point $s \in \mathbb{R}$, are defined by

$$
\gamma_{ \pm}(\mu ; s)=\lim \sup (\inf )_{\epsilon \rightarrow 0} \frac{\log \mu((s-\epsilon, s+\epsilon))}{\log \epsilon} .
$$


Definition 8 The upper (lower) correlation dimensions $D_{2}^{ \pm}(\mu)$ of a measure $\mu$, are defined by

$$
D_{2}^{ \pm}(\mu)=\lim \sup (\inf )_{\epsilon \rightarrow 0} \frac{\log \int d \mu(s) \mu([s-\epsilon, s+\epsilon])}{\log \epsilon} .
$$

Theorem $2([3],[4])$ The electrostatic dimensions $d(\mu ; s)$ and $D_{2}(\mu)$ coincide with the lower dimensions: $\gamma_{-}(\mu ; s)=d(\mu ; s), D_{2}^{-}(\mu)=D_{2}(\mu)$.

As a matter of fact, Theorem 2 is a consequence of a general theorem on Mellin (and Laplace) transforms that can be found more or less explicitly in the literature. A sketch of its proof will be helpful:

Theorem 3 Let $m(u)$ a Stieltjes measure on $[0,1]$, such that $m(0)=0, m(1)<$ $\infty$, and let $M_{[0,1]}(m ; z)$ be the Mellin-Stieltjes transform of $m$ :

$$
M_{[0,1]}(m ; z):=\int_{0}^{1} d m(u) u^{-z} .
$$

Let $\delta(m)$ be the divergence abscissa of $M_{[0,1]}(m ; z)$. Clearly, $\delta(m) \geq 0$. Moreover,

$$
\lim \inf _{\epsilon \rightarrow 0} \frac{\log m(\epsilon)}{\log \epsilon}=\delta(m) .
$$

Proof: Notice the different definition adopted for the definition of the M.T. of a measure on $[0,1]$, when compared to the usual eq. (6). Take $x \in \mathbb{R}_{+}, x<\delta(m)$. Then, $M_{[0,1]}(m ; x)$ is finite, smaller than a positive constant $B$. Here and in the following the letter $B$ will indicate various constants appearing in the proofs. This implies the chain of inequalities $B \geq \int_{0}^{\varepsilon} d m(u) u^{-x} \geq \varepsilon^{-x} \int_{0}^{\varepsilon} d m(u)=\varepsilon^{-x} m(\varepsilon)$ for all $\varepsilon$. As a consequence, $\frac{\log m(\epsilon)}{\log \epsilon} \geq x+\frac{\log B}{\log \epsilon}$. Letting $l$ be the liminf in eq. (13), this implies $l \geq x$ for any $x<\delta(m)$. Let us now show the reverse inequality. Because of the definition of lower limit, for any $\eta>0$, there exist $\varepsilon_{\eta}$ such that, for any $\varepsilon<\varepsilon_{\eta}<1, m(\varepsilon) \leq \varepsilon^{l-\eta}$. By a standard Laplace-type estimate we can write:

$M_{[0,1]}(m ; x)=\int_{0}^{\varepsilon_{\eta}} d m(u) u^{-x}+\int_{\varepsilon_{\eta}}^{1} d m(u) u^{-x} \leq\left. u^{-x} m(u)\right|_{0^{+}} ^{\varepsilon_{\eta}}+x \int_{0}^{\varepsilon_{\eta}} d u u^{-x-1} m(u)+B \varepsilon_{\eta}^{-x}$.

The last term at r.h.s. is obviously finite. Let us consider the first term. The contribution at $\varepsilon_{\eta}$ is also finite. Moreover, $u^{-x} m(u) \leq u^{-x+l-\eta}$, so that $\lim _{u \rightarrow 0^{+}} u^{-x} m(u)$ is null when $x<l-\eta$. For the same reason, under this condition the remaining integral term is also finite, and so is $M(m ; x)$. This holds for all $\eta$, and therefore the divergence abscissa $\delta(m)$ is larger than, or equal to, $l$.

Proof of Thm. 2. It follows from Thm. 3. For the local dimension, the Stieltjes measure is $m_{s}(u):=\mu([s-u, s+u])$, and

$$
M_{[0,1]}\left(m_{s} ; z\right)=\mathcal{G}(\mu ; s, z) .
$$


Because of this, the proof is closely related to Theorem 6.4 in [14] - albeit we employ a slightly different technique. For the correlation dimension, one considers the correlation measure $\Omega(\mu ; u)$ defined via

$$
\Omega(\mu ; u):=\iint_{|s-r| \leq u} d \mu(s) d \mu(r),
$$

and the result follows from

$$
M_{[0,1]}(\Omega ; z)=\mathcal{E}(\mu ; z) .
$$

Remark 1 Formula (14) above shows that the correlation dimension can be equivalently considered as the local dimension, at the point zero, of the correlation measure associated with $\mu$. Therefore, the results that we shall obtain for the local dimensions will immediately extend mutatis mutandis to the correlation dimension.

\section{Long time limits: Laplace-like results}

The well known asymptotic behaviour of the Fourier transform of a measure can be also seen as a particular case of the general Thm. 3. For this, we need a close analogue of this latter:

Theorem 4 Let $m(u)$ a Stieltjes measure on $[1, \infty)$, and let the Mellin-Stieltjes transform of $m, M_{[1, \infty)}(m ; z)$, be defined as:

$$
M_{[1, \infty)}(m ; z):=\int_{1}^{\infty} d m(u) u^{z-1} .
$$

Let $\delta(m)$ be its divergence abscissa. Put $\alpha(m):=\min \{\delta(m), 1\}$. Let $C_{1}(m ; r)$ be the one-sided, lower bounded Cesaro average, defined as

$$
C_{1}(m ; r):=\frac{1}{r} \int_{1}^{r} d m(u)
$$

and finally let $L:=\limsup _{r \rightarrow \infty} \log C_{1}(m ; r) / \log r$. Then,

$$
-\delta(m) \leq L \leq-\alpha(m) .
$$

Proof: We sketch only the differences with the proof of Thm. 3. The first part of the proof differs only in the fact that, in order to get the inequality $r^{x} C_{1}(m ; r) \leq B$ one must require that $x<\delta(m)$ and, in addition, $x \leq 1$. In so doing, the second inequality in (17) follows. The second part of the proof is basically unchanged, with the sole substitution of liminf with limsup, and leads to the first inequality in the thesis. 
Remark 2 It is easy to see that the thesis can be equivalently stated in terms of the usual Cesaro average $\mathcal{C}(f ; t)$, when the integral of $d m(u)=f(u) d u$ between zero and one can be controlled.

This theorem highlights the relation between the asymptotics of the Fourier transform and the correlation dimension:

Theorem 5 Let $d m(t)=\left|\mathcal{J}_{0}(\mu ; t)\right|^{2} d t$, so that $C_{1}(m ; t)$ is the Cesaro average of the square modulus of the Fourier transform of the measure $\mu$. Then,

$$
\limsup _{t \rightarrow \infty} \log C_{1}(m ; t) / \log t=-D_{2}(\mu) .
$$

Proof: We need to compute the Mellin transform

$$
M_{[1, \infty)}(m ; z)=\int_{1}^{\infty} d m(t) t^{z-1}=\lim _{T \rightarrow \infty} \int_{1}^{T} d t t^{z-1} \iint d \mu(r) d \mu(s) e^{-i t(r-s)} .
$$

This can be also written as

$$
M_{[1, \infty)}(m ; z)=\lim _{T \rightarrow \infty} \iint d \mu(r) d \mu(s) \frac{1}{|r-s|^{z}} \int_{|r-s|}^{|r-s| T} d u u^{z-1} e^{-i u},
$$

thanks to a change of variables, to the symmetry of the domain of integration, and under the condition that $\Re(z)<D_{2}(\mu)$, so that the integrand of the triple integral is summable, to apply Fubini theorem. Now, observe that when $0<\Re(z)<1$ the integral with respect to $d u$ in the above expression is a bounded function of $|r-s|$ and $T$. Then, $\frac{1}{|r-s|^{z}} \int_{|r-s|}^{|r-s| T} d u u^{z-1} e^{-i u}$ is uniformly bounded (with respect to $T$ ) in the $L^{1}$ norm, and the limit can be taken into the double integral, to get

$$
M_{[1, \infty)}(m ; z)=\iint d \mu(r) d \mu(s) \frac{1}{|r-s|^{z}} \int_{|r-s|}^{\infty} d u u^{z-1} e^{-i u} .
$$

When $|r-s|$ tends to zero, the inner integral tends continuously to the Mellin transform of the exponential function of imaginary argument, $M\left(e^{i t}, z\right)=\Gamma(z) e^{i \pi z / 2}$ : as a consequence, divergence is dictated by the kernel $\frac{1}{|r-s|^{z}}$ so that $D_{2}(\mu)$ is the divergence abscissa of the Mellin transform $M_{[1, \infty)}(m ; z)$. To conclude the proof, apply Thm. 4 .

Remark 3 This theorem is fully equivalent to the analysis of Last, Lemma 5.2 of [20], and the remark just following. Scrutiny of these proofs shows their equivalence, via Thm. 4, to the proofs of Thm. 2 in [3], [4]. Our proof of Thm. 2 masters differently one of the inequalities, in a way that is closer to the standard usage in Laplace transforms. 


\section{Local Properties of the Measure and Asymp- totic Decay of F-B. functions.}

We now start the analysis of the asymptotic behaviour of the Cesaro averages $\overline{\mathcal{J}}_{n}(\mu ; t)$. We shall find that it is related to the local properties of the measure $\mu$ at zero. This will entail notational simplification with respect to the previous section, by dropping the explicit reference to the point zero. A movable local analysis will be reintroduced starting from Sect. 16 .

We start by re-writing the function $M_{n}(\mu ; z)$, eq. (6), in a convenient way. Notice that throughout this paper we shall write $z=x+i y$, with $x$ and $y$ real. Firstly, we observe that the integral

$$
I_{n}(\mu ; x)=\int d \mu(s) \int_{0}^{\infty} d t\left|t^{x-1} p_{n}(\mu ; s) \frac{\sin t s}{t s}\right|
$$

is convergent, when $x$ belongs to a suitable interval. In fact, we can split the inner integral in two parts, obtaining

$$
\begin{aligned}
I_{n}(\mu ; x) & \leq \int d \mu(s)\left|p_{n}(\mu ; s)\right|\left[\int_{0}^{\frac{\pi}{2 s \mid}} d t t^{x-1}+\int_{\frac{\pi}{2 s \mid}}^{\infty} d t t^{x-1} \frac{1}{t|s|}\right] \\
& =\left[\frac{1}{x}\left(\frac{\pi}{2}\right)^{x}+\frac{1}{1-x}\left(\frac{\pi}{2}\right)^{x-1}\right] \int d \mu(s)\left|p_{n}(\mu ; s)\right||s|^{-x},
\end{aligned}
$$

where we are forced to restrict $x$ to $(0,1)$, the r.h.s. being infinite otherwise. Therefore, if $0<x<1$ and the integral

$$
\mathcal{G}_{n}(\mu ; x)=\int d \mu(s) \frac{\left|p_{n}(\mu ; s)\right|}{|s|^{x}},
$$

is convergent, so is $I_{n}(\mu ; x)$. The integral $\mathcal{G}_{0}(\mu ; x)$ has been introduced previously: it is the generalized electrostatic potential at the point zero. It divergence abscissa, defines the local dimension at the point $s=0$ [27]. This latter is certainly larger than, or equal to zero, since $\mu$ is a probability measure, and $p_{n}(\mu ; s)$ are its orthogonal polynomials. We now need to adapt the definitions to let these latter play a rôle:

Definition 9 The electrostatic local dimensions of $\mu$ at zero, $d_{n}(\mu)$, are the divergence abscissas of the integrals $\mathcal{G}_{n}(\mu ; z)$,

$$
d_{n}(\mu):=\sup \left\{x \in \mathbb{R} \text { s.t. } \mathcal{G}_{n}(\mu ; x)<\infty\right\} .
$$

Remark 4 Because of Def. 5 , this is equivalent to say that $d_{n}(\mu)$ is the local dimension, at zero, of the measure $\nu=\left|p_{n}\right| \mu: d_{n}(\mu)=d\left(\left|p_{n}\right| \mu ; 0\right)$.

Definition 10 The asymptotic exponents $\alpha_{n}(\mu)$ are the divergence abscissas of the integrals $I_{n}(\mu ; z)$ :

$$
\alpha_{n}(\mu):=\sup \left\{x \in \mathbb{R} \text { s.t. } I_{n}(\mu ; x)<\infty\right\} .
$$


Asymptotic exponents and local dimensions are related as follows:

Proposition 1 If $p_{n}(0) \neq 0$, the dimension $d_{n}(\mu)$ is equal to $d_{0}(\mu)$. If $p_{n}(0)=0$, the dimension $d_{n}(\mu)$ is equal to $d_{0}(\mu)+1$. Moreover,

$$
\alpha_{n}(\mu)=\min \left\{d_{n}(\mu), 1\right\}
$$

Proof. Let $x \geq 0, a>0$.

$$
\mathcal{G}_{n}(\mu ; x)=\int_{|s|<a} d \mu(s) \frac{\left|p_{n}(\mu ; s)\right|}{|s|^{x}}+\int_{|s| \geq a} d \mu(s) \frac{\left|p_{n}(\mu ; s)\right|}{|s|^{x}}
$$

The second integral defines an analytic function. In fact, it can be bounded by

$$
\frac{1}{|a|^{x}} \int d \mu(s)\left|p_{n}(\mu ; s)\right| \leq \frac{1}{|a|^{x}}\left(\int d \mu(s)\left|p_{n}(\mu ; s)\right|^{2}\right)^{1 / 2}=\frac{1}{|a|^{x}},
$$

because $\mu$ is a probability measure, and orthogonal polynomials are normalized. Consider now the first integral in $(23)$. If $p_{n}(0) \neq 0$, choose $a$ such that on $[-a, a]$ $p_{n}(\mu ; s)$ is strictly different from zero. Let $m=\min \left\{\left|p_{n}(\mu ; s)\right|,|s|<a\right\}, M=$ $\max \left\{\left|p_{n}(\mu ; s)\right|,|s|<a\right\}$. We have

$$
m \int_{|s|<a} d \mu(s)|s|^{-x} \leq \int_{|s|<a} d \mu(s)\left|p_{n}(\mu ; s)\right||s|^{-x} \leq M \int_{|s|<a} d \mu(s)|s|^{-x}
$$

and the first part of the thesis follows. When $p_{n}(0)=0$, recall that zeros of orthogonal polynomials are always simple, so that: $p_{n}(\mu ; s)=s q_{n-1}(s)$, with $q_{n-1}(0) \neq 0$, and apply the same reasoning.

The inequality $\alpha_{n}(\mu) \geq \min \left\{d_{n}(\mu), 1\right\}$ follows upon consideration of eq. (19). The reverse inequality can be obtained from

$$
\begin{gathered}
I_{n}(\mu ; x) \geq \int d \mu(s)\left|p_{n}(\mu ; s)\right| \int_{\frac{\pi}{2|s|}}^{\infty} d t t^{x-1}|\sin c(t|s|)|= \\
=\int d \mu(s) \frac{\left|p_{n}(\mu ; s)\right|}{|s|^{x}} \int_{\frac{\pi}{2}}^{\infty} d u u^{x-2}|\sin (u)|,
\end{gathered}
$$

that shows that if one of the integrals at r.h.s. is divergent, so is $I_{n}(\mu ; x)$.

Remark 5 The first case considered in the proposition above, $p_{n}(0) \neq 0$, is obviously typical. On the other hand, $p_{n}(0)=0$ happens for instance when the measure is symmetric with respect to zero, and $n$ is odd, $p_{n}(-x)=-p_{n}(x)$ : the divergence abscissa $d_{n}(\mu)$ is then infinite. But in this case the Cesaro average we are studying is null, and our analysis is empty. A remedy is then to employ a one-sided Cesaro average, between 0 and $t$.

The asymptotic exponents $\alpha_{n}(\mu)$ deserve their name in virtue of the following fundamental theorem. 
Theorem 6 Let $\alpha_{n}(\mu)$ be the asymptotic exponents in Def. 10. For all $x$ such that $x<\alpha_{n}(\mu)$, when $t \rightarrow \infty$, the asymptotic estimate $\overline{\mathcal{J}}_{n}(\mu ; t)=o\left(t^{-x}\right)$ holds.

Proof. Because of equation (4), one writes

$$
t^{x} \overline{\mathcal{J}}_{n}(\mu ; t)=\int d \mu(s) t^{x} \operatorname{sinc}(t s) p_{n}(\mu ; s)=\int \frac{d \mu(s)}{|s|^{x}}|s t|^{x} \sin c(|t s|) p_{n}(\mu ; s) .
$$

Let $x \in[0,1]$. Then the function $u \rightarrow u^{x} \operatorname{sinc}(u)$ is bounded by a constant $B$ on $\mathbb{R}$. Therefore, $\left.|| s t\right|^{x} \operatorname{sinc}(|t s|) p_{n}(\mu ; s)|\leq B| p_{n}(\mu ; s) \mid$. Since $x<\alpha_{n}(\mu)$, this latter function is integrable with respect to the measure $d \mu(s) /|s|^{x}$. Then, the dominated convergence theorem permits to take the limit for infinite $t$ inside the integral sign, so that $t^{x} \overline{\mathcal{J}}_{n}(\mu ; t) \rightarrow 0$.

\section{Analytic Representation of the M.T.}

In this section we investigate the analyticity properties of the Mellin transform $M_{n}(\mu ; z)$. The considerations of Sect. 5 can be regarded as preparatory work to the following: when $x=\Re(z)<\alpha_{n}(\mu)$, it is legitimate to exchange the order of the integrals defining $M_{n}(\mu ; z)$, to obtain:

Proposition 2 The integral representation

$$
M_{n}(\mu ; z)=\int_{0}^{\infty} d t t^{z-1} \int d \mu(s) p_{n}(\mu ; s) \frac{\sin t s}{t s}
$$

defines an analytic function in the domain $0<\Re(z)<\alpha_{n}(\mu)$. This function can be also expressed as

$$
M_{n}(\mu ; z)=H(z) G_{n}(\mu ; z),
$$

where $H(z)$ is the analytic continuation of the Mellin transform of $\operatorname{sinc}(\cdot)$,

$$
H(z)=\Gamma(z-1) \sin \left[\frac{\pi}{2}(z-1)\right]
$$

and where

$$
G_{n}(\mu ; z)=\int d \mu(s) \frac{p_{n}(\mu ; s)}{|s|^{z}}
$$

Proof: Since

$$
\int_{0}^{\infty} d t\left|t^{z-1} \int d \mu(s) p_{n}(\mu ; s) \frac{\sin t s}{t s}\right| \leq I_{n}(\mu ; x)<\infty
$$

the function $t \rightarrow t^{z-1} \overline{\mathcal{J}}_{n}(\mu ; t)$ belongs to $L^{1}([0, \infty], d t)$ and the first part of the thesis follows. Moreover, Fubini theorem applies: changing the order of integration in eq. (25) then provides the formulae (26), (27),(28). 
Remark 6 The function $H(z)$ is purely kinematical, and does not depend on the measure $\mu$. It is a meromorphic function, with simple poles at $-2 k, k=0,1, \ldots$, of residuals $\rho_{k}=(-1)^{k}$. The pole at zero is related to the $O(1)$ behavior of $\sin (t) / t$ for small $t$. The second term in eq. $(26), G_{n}(\mu ; z)$, explicitly calls in cause the short scale properties of the measure $\mu$ at zero. Because of this term, we see that $M_{n}(\mu ; z)$ is convergent, for $\Re(z) \in[0,1)$, if and only if $G_{n}(\mu ; z)$ is such. Furthermore, if $d_{n}(\mu)$ is larger than one, $M_{n}(\mu ; z)$ can be analytically continued for $\Re(z)>1$ via eq. (26) until the first singularity of $\mathcal{G}_{n}(\mu ; z), d_{n}(\mu)$. Of course, this analytical continuation does not imply that the original Mellin transform integral is convergent in Lebesgue sense. In Sect. 9 we shall employ improper Riemann integrals to extend the domain of convergence.

\section{Inversion of M.T. and Asymptotic Properties}

We can now return to the analysis of the asymptotic behaviour of the integral (4). The poles of $H(z)$, possessing a non-positive real part, are related to the shorttime behaviour of $\overline{\mathcal{J}}_{n}(\mu ; t)$. Indeed, because of the orthogonality property of the polynomials $p_{n}$ with respect to $\mu$, it is easy to see that some of these poles are cancelled by zeros of $G_{n}(\mu ; z)$ :

$$
G_{n}(\mu ;-2 k)=0, \text { for } 2 k<n .
$$

We can use this information to obtain a precise asymptotic of $\overline{\mathcal{J}}_{n}(\mu ; t)$ for small times.

On the contrary, large times are governed by the interplay between the averaging procedure and the singularities of $G_{n}(\mu ; z)$ at, and past, the divergence abscissa $d_{n}(\mu)$. In certain notable cases, as for linear Iterated Function Systems, discussed in the next paper, these singularities can be mastered, and a precise asymptotic information obtained. In this section and in the next, we develop the techniques to achieve this control. The same techniques, in the absence of any information on the singularities provide nonetheless estimates from above on the decay of $\overline{\mathcal{J}}_{n}(\mu ; t)$.

Formally, the Mellin transform of any function $f$ can be seen as a Fourier transform in logarithmic time $\tau=\log t$ :

$$
\mathcal{M}(f ; x+i y)=\int_{-\infty}^{\infty} d \tau e^{x \tau} f\left(e^{\tau}\right) e^{i y \tau} .
$$

We apply this equality to $f(t)=\overline{\mathcal{J}}_{n}(\mu ; t)$, and set

$$
h_{n}(x, \tau):=e^{x \tau} \overline{\mathcal{J}}_{n}\left(\mu ; e^{\tau}\right) .
$$

Then,

$$
\left.M_{n}(\mu ; x+i y)\right)=\mathcal{F}\left(h_{n}(x, \tau)\right)(-y),
$$

where, as usual, $\mathcal{F}$ indicates the Fourier transform. So far, this is purely formal. We now make this precise. 
Proposition 3 For $0<x<\alpha_{n}(\mu), h_{n}(x, \tau)$ belongs to $L^{1}(\mathbb{R}, d \tau)$ and therefore $M_{n}(\mu ; x+i y)$ exists and belongs to $\mathcal{C}_{0}(\mathbb{R}, y)$, the set of continuous functions (of the real variable $y$ ) vanishing at infinity.

Proof. Because of Proposition 1, and of Fubini theorem,

$$
\begin{gathered}
\int_{-\infty}^{\infty} d \tau\left|h_{n}(x, \tau)\right|=\int_{-\infty}^{\infty} d \tau\left|e^{x \tau} \int d \mu(s) p_{n}(\mu ; s) \operatorname{sinc}\left(e^{\tau} s\right)\right| \leq \\
\leq \int_{0}^{\infty} d t \int d \mu(s)\left|p_{n}(\mu ; s) t^{x-1} \operatorname{sinc}(s t)\right|=I_{n}(\mu ; x)<\infty
\end{gathered}
$$

Lemma 1 For $x<\alpha_{n}(\mu), G_{n}(\mu, x+i y)$ is a bounded, continuous function of $y$.

Proof. Clearly, $\left|G_{n}(\mu, x+i y)\right| \leq G_{n}(\mu ; x)<\infty$, which also allows to apply the dominated convergence theorem.

Now, we present sufficient conditions for $\left.M_{n}(\mu ; x+i y)\right)$ to belong to an integrability class.

Lemma 2 For $0<x<\min \left(\alpha_{n}(\mu), \frac{1}{2}\right)$, the function $\left.M_{n}(\mu ; x+i y)\right)$ belongs to $L^{1}(\mathbb{R}, d y) ;$ For $0<x<\alpha_{n}(\mu)$, the function $\left.M_{n}(\mu ; x+i y)\right)$ belongs to $L^{2}(\mathbb{R}, d y)$.

Proof. Because of Stirling formula $\Gamma(z)=e^{-z} e^{\left(z-\frac{1}{2}\right) \log z} \sqrt{2 \pi}\left[1+O\left(\frac{1}{|z|}\right)\right]$ for $z \rightarrow$ $\infty,|\arg z|<\pi$, we have that

$$
|\Gamma(x+i y-1)| \sim \sqrt{2 \pi}|y|^{-\frac{3}{2}+x} e^{-\frac{\pi}{2}|y|} \quad \text { for }|y| \rightarrow \infty .
$$

Therefore, the asymptotic behaviour of $H(x+i y)$, when $y \rightarrow \pm \infty$, is

$$
|H(x+i y)| \simeq|y|^{-\frac{3}{2}+x}\left(1+o\left(\frac{1}{|y|}\right)\right) .
$$

In addition, because of Lemma $1, G_{n}(\mu ; x+i y)$ is bounded in $y$ for fixed $x$. Because of the previous lemma, and of Proposition 3, $H(x+i y)$ is also continuous. Then, it is integrable in $y$ when $x<\frac{1}{2}$, and square summable for $x<1$.

We can therefore take the inverse Fourier transform of $M_{n}(\mu ; x+i y)$ :

Lemma 3 For $0<x<\min \left(\alpha_{n}(\mu), \frac{1}{2}\right)$,

$$
h_{n}(x, \tau)=\frac{1}{2 \pi} \int_{-\infty}^{\infty} d y e^{-i \tau y} M_{n}(\mu ; x+i y) .
$$

Proof. In the $x$-range considered, the inverse transform of $M_{n}(\mu ; x+i y)$ exists, and belongs to $\mathcal{C}_{0}(\mathbb{R}, \tau)$. It then coincides with the continuous function $h_{n}(x, \tau)$.

This result allows us to obtain a (weaker) variant of Thm. 6 : 
Theorem 7 For all $x$ such that $x<\min \left(\alpha_{n}(\mu), \frac{1}{2}\right)$, the function $\overline{\mathcal{J}}_{n}(\mu ; t)$ can be written as: $\overline{\mathcal{J}}_{n}(\mu ; t)=t^{-x} s_{n}(x ; t)$ where $s_{n}(x ; t)$ belongs to $\mathcal{C}_{0}\left(\mathbb{R}_{+}, t\right)$.

Proof. Because of Lemma $7, h_{n}(x, \tau) \in \mathcal{C}_{0}(\mathbb{R}, \tau)$. Returning to linear time $t$, and using eq. (31) gives the thesis.

The fact that in the above Theorem the value of $x$ is bound to be smaller than one half, even when $\alpha_{n}(\mu)$ is not, is a limitation due to the technique, rather than to the nature of the problem, as it appears from Thm. 6. First of all, the range where the $L^{1}$ property of $M_{n}(x+i y)$ holds can sometimes be extended. We shall see this in Sect. 14. Also, a few other instructive ways exist to find a superior limit to the decay exponent. In the next section, Sect. 8, we follow a $L^{2}$ technique originally developed by Makarov, that permits to obtain an asymptotic decay in weak form. Then, in Sect. 9, we employ a different technique to obtain the asymptotic decay in strong form.

\section{$8 L^{2}$ representation of the asymptotic decay}

The asymptotic decay of $\overline{\mathcal{J}}_{n}(\mu ; t)$ can be obtained also in the full range $0<\alpha_{n}(\mu)<$ 1 following the $L^{2}$ technique of Makarov [5]:

Theorem 8 For all $x$ such that $x<\alpha_{n}(\mu), \overline{\mathcal{J}}_{n}(t)$ can be written as $\overline{\mathcal{J}}_{n}(t)=$ $t^{-x} s_{n}(x, t)$, where $s_{n}(x, t)$ belongs to $L^{2}\left((0, \infty), t^{-1} d t\right)$.

Proof. We must consider the sequence of paths $\gamma_{N}$ defined as follows. They consist of the straight vertical segment $x+i y$, with $x<\frac{1}{2}, y \in[-N, N]$, the horizontal segment from $x+i N$ to $\bar{x}+i N$, with $\min \left(\alpha_{n}(\mu), 1\right)>\bar{x}>\frac{1}{2}$ and the two remaining segments needed to form a rectangle in complex plane. Then, let us consider the function $m(\tau ; z):=M_{n}(\mu ; z) e^{-\tau z}$, with $\tau \in \mathbb{R}_{+}$where the dependence on $n$ and $\mu$ is left implicit. This function is analytic in the the strip $0<\Re(z)<\min \left(\alpha_{n}(\mu), 1\right)$ and therefore, its contour integral over $\gamma_{N}$ is null. Moreover, it is easy to see that the integral on the horizontal pieces go to zero, as $N$ tends to infinity, thanks to (33). Taking this limit, the integral over the left vertical segment at $x$ tends to $2 \pi i$ times $h_{n}(x, \tau) e^{-\tau x}$, which is nothing else than $2 \pi i \overline{\mathcal{J}}_{n}\left(\mu ; e^{\tau}\right)$, independently of $x$. Therefore, also the integral on the right vertical segment, at abscissa $\bar{x}$, tends to the same limit. Expanding the integral, this means that, pointwise in $\tau$,

$$
\overline{\mathcal{J}}_{n}\left(\mu ; e^{\tau}\right)=\frac{1}{2 \pi i} e^{-\tau \bar{x}} \lim _{N \rightarrow \infty} \int_{-N}^{N} d y e^{-i \tau y} M_{n}(\mu ; \bar{x}+i y) .
$$

Consider now the integrals

$$
I_{N}=\int_{-N}^{N} d y e^{-i \tau y} M_{n}(\mu ; \bar{x}+i y) .
$$

Now, because of Lemma 2 , when $\bar{x}$ belongs to the range considered, the function $M_{n}(\mu ; \bar{x}+i y)$ belongs to $L^{2}$, and therefore, the $L^{2}$ limit of the integral (36) is a 
Fourier-Plancherel transform, call it $q(\bar{x} ; \tau)$. Since there exists a subsequence $N_{k}$ such that $I_{N_{k}}$ converges almost everywhere to $q(\bar{x} ; \tau)$, we see immediately that

$$
\overline{\mathcal{J}}_{n}\left(\mu ; e^{\tau}\right)=e^{-\tau \bar{x}} q(\bar{x} ; \tau),
$$

where $q(\bar{x} ; \tau)$ belongs to $L^{2}((-\infty, \infty), d \tau)$. Returning now to linear time, $t=e^{\tau}$, we get $\overline{\mathcal{J}}_{n}(\mu ; t)=t^{-\bar{x}} s_{n}(\bar{x} ; t)$, where $s_{n}(\bar{x} ; t)$ belongs to $L^{2}\left((0, \infty), t^{-1} d t\right)$.

Remark 7 We have outlined this technique for its importance when the integration contour can be extended to enclose the singularities of $M_{n}(\mu ; z)$. The result of the previous Theorem could infact be obtained also from Thm. 6 that shows that $h_{n}(x, \tau)$ belongs to $L^{p}(\mathbb{R}, d \tau)$ for all $p>0$ and $x<\alpha_{n}(\mu)$.

\section{Strong form of the asymptotic decay}

In this section, we apply a different technique to show that the strong asymptotic form of $\overline{\mathcal{J}}_{n}(\mu ; t)$ can be obtained also in the full exponent range [0,1], and possibly beyond it. In fact, let $g(t)$ be a Riemann integrable real function, $\bar{g}(t)$ be its Cesaro average, $\bar{g}(t):=\mathcal{C}(g ; t)$ and let $\mathcal{M}(g ; z, T)$ be the truncated Mellin transform

$$
\mathcal{M}(g ; z, T):=\int_{0}^{T} d t g(t) t^{z-1} .
$$

Define analogously $\mathcal{M}(\bar{g} ; z, T)$. When the improper Riemann integral $\mathcal{M}(g, z, \infty)$ exists, it defines the Mellin transform $\mathcal{M}(g, z)$. A simple calculation shows that

Lemma 4 For any continuous function $g$ and any $x \neq 1, x>0$ :

$$
\mathcal{M}(\bar{g} ; x, T)=\frac{1}{x-1}\left[T^{x} \bar{g}(T)-\mathcal{M}\left(g_{e} ; x, T\right)\right]
$$

where $g_{e}(t):=(g(t)+g(-t)) / 2$

Proof: The equation above follows simply by integration by parts in the definition of $\mathcal{M}(\bar{g} ; x, T)$.

This lemma can be used to obtain the asymptotic behaviour of $\bar{g}(t)$ :

Theorem 9 Suppose that there exists $q \in \mathbb{R}_{+}$such that the Mellin transforms $\mathcal{M}(\bar{g}, q)$ and $\mathcal{M}\left(g_{e}, q\right)$ exist as convergent improper Riemann integrals. Then,

$$
\bar{g}(t)=o\left(t^{-q^{\prime}}\right)
$$

for all $q^{\prime}<q$.

Proof: Clearly, if the improper integrals defining $\mathcal{M}(\bar{g}, q)$ and $\mathcal{M}\left(g_{e}, q\right)$ converge, so must $T^{q} \bar{g}(T)$ do for $T \rightarrow \infty$, because of eq. (39) and therefore the lemma follows.

We apply this Theorem to $g(t)=\mathcal{J}_{n}(\mu ; t)$, the non-averaged F-B. functions. 
Proposition 4 For $\Re(z)<\alpha_{n}(\mu)$, the Mellin transform of $\mathcal{J}_{n}(\mu ; t)$ exists as a convergent improper Riemann integral, and it can be represented as

$$
\mathcal{M}\left(\mathcal{J}_{n} ; z\right)=\int_{0}^{\infty} d t t^{z-1} \mathcal{J}_{n}(\mu ; t)=\Gamma(z) e^{i \frac{\pi}{2} z} G_{n}(\mu ; z) .
$$

Proof. The following limit, when it exists, defines the Mellin transform in Riemann sense:

$$
\mathcal{M}\left(\mathcal{J}_{n} ; z\right)=\lim _{T \rightarrow \infty} \int_{0}^{T} d t t^{z-1} \int d \mu(s) e^{-i t s} p_{n}(\mu ; s) .
$$

In fact, when $\Re(z)>0$ integration at the leftmost limit is controlled, since $\mathcal{J}_{n}(\mu ; t)$ are bounded. Moreover, the double integral inside the limit is also an absolutely convergent Lebesgue integral - therefore, the order of integration in eq. (41) can be inverted, and a change of variables performed, to get

$$
\mathcal{M}\left(\mathcal{J}_{n} ; z\right)=\lim _{T \rightarrow \infty} \int d \mu(s) p_{n}(\mu ; s) \frac{1}{|s|^{z}} \int_{0}^{|s| T} d u u^{z-1} e^{i u} .
$$

We now move the limit within the integral with respect to $d \mu$, in force of the dominated convergence theorem. Let $h_{T}(s)$ be the integrand in eq. (42):

$$
h_{T}(s):=p_{n}(\mu ; s) \frac{1}{|s|^{z}} \int_{0}^{|s| T} d u u^{z-1} e^{i u} .
$$

It is a matter of fact that there exists a summable majorant $h$ of $h_{T}$ : this can be proven as follows. In the first place the integral $I(a):=\int_{0}^{a} d u u^{z-1} e^{i u}$ is a continuous function of the upper integration limit $a$. Furthermore, as $a$ tends to infinity, for all $z$ such that $0<\Re(z)<1$, the integral tends to a finite limit that is the Mellin transform of the exponential function of imaginary argument, $M\left(e^{i t}, z\right)=\Gamma(z) e^{i \pi z / 2}$. Consequently, $|I(a)|$ is bounded by a constant $B$, that depends only on $z$, and so is the companion integral in eq. (43). Summing all this together, we have

$$
\left|h_{T}(s)\right| \leq B \frac{\left|p_{n}(\mu ; s)\right|}{|s|^{z}}:=h(s) .
$$

Since $\Re(z)<d_{n}(\mu), h(s)$ is summable, and we can conclude, letting $M\left(e^{i t}, z\right)$ appear in eq. (42), that takes the final form (40).

Notice that replacing $e^{-i t s}$ by $\operatorname{sinc}(t s)$ in eq. (41) and following, we obtain an extension of the range of convergence of the integral representation, and of validity of the formulae in Proposition 2:

Proposition 5 For $\Re(z)<\min \left\{d_{n}(\mu), 2\right\}$, the Mellin transform of $\overline{\mathcal{J}}_{n}(\mu ; t)$ exists as a convergent improper Riemann integral, and it can be represented as in Proposition 2, eqs. (26,27,28). 
Proof. We need only to observe that the integral $I(a)$ in the proof of Proposition 4 becomes here $I(a):=\int_{0}^{a} d u u^{z-1} \operatorname{sinc}(u)$, whose modulus is a bounded function of $a$ for all $0<\Re(z) \leq 2$. Compare also with the proof of Thm. 5

Remark 8 We can now obtain a different proof of Thm. 6:

Alternative proof of Thm. 6. It follows from the previous proposition that the Mellin transforms of $\overline{\mathcal{J}}_{n}(\mu ; t)$ and $\mathcal{J}_{n}(\mu ; t)$ have the same behaviour in $(0,1)$ : as improper Riemann integrals, they are either both convergent, or divergent. Then, the theorem follows from Thm. 9.

Remark 9 Proposition 5 above opens the possibility that an extension of the range of asymptotic decay of $\overline{\mathcal{J}}_{n}(\mu ; t)$ might be found also when $1<d_{0}(\mu)<2$, that is, beyond the reach of Thm. 6. Indeed, the result of Proposition 4 do not exclude that the Mellin transform of $\mathcal{J}_{n}(\mu ; t)$ might exist as an improper Riemann integral also when $\Re(z)>1$, of course when $\Re(z)<d_{0}(\mu)$. This is the case of the example worked out in Appendix.

\section{Decay of quadratic amplitudes: asymptotic ex- ponents}

We now study the quadratic amplitudes $A_{n m}(\mu ; t)$. Part of this theory is merely a two dimensional generalization of the previous sections, so that we shall be concise in the exposition. To the contrary, new features originating from positivity are to be found in the diagonal $n=m$ case. From the definition (5), the amplitudes can be written as:

$$
A_{n m}(\mu ; t)=\iint d \mu(r) d \mu(s) p_{n}(\mu ; r) p_{m}(\mu ; s) \operatorname{sinc}(t(r-s)) .
$$

It is evident from eq. (44) that $A_{n m}$ are real quantities.

We start by computing the integral representation of the Mellin transform of the amplitudes $A_{n m}(\mu ; t)$, that we have denoted $M_{n m}(\mu ; z)$. The $\mu$ dependence of the orthogonal polynomials $p_{n}(\mu ; s)$ and of other quantities will be left implicit at times, not to overburden the notation. Because of eq. (44), this can be written

$$
M_{n m}(\mu ; z)=\int_{0}^{\infty} d t t^{z-1} \iint d \mu(r) d \mu(s) p_{n}(r) p_{m}(\mu ; s) \operatorname{sinc}(t(r-s)) .
$$

Before tackling $M_{n m}(\mu ; z)$ directly, we consider a companion integral $I_{n m}(z)$ to (45), as we did in Sect. 5. We put, as usual, $z=x+i y$, with $x, y \in \mathbb{R}$ :

$$
\begin{aligned}
I_{n m}(z) & :=\iint d \mu(r) d \mu(s) \int_{0}^{\infty} d t\left|t^{z-1}\right|\left|p_{n}(r) p_{m}(\mu ; s)\right||\operatorname{sinc}(t|r-s|)|= \\
& =\iint d \mu(r) d \mu(s) \frac{\left|p_{n}(r) p_{m}(\mu ; s)\right|}{|r-s|^{x}} \int_{0}^{\infty} d \xi \xi^{x-1}|\operatorname{sinc}(\xi)| .
\end{aligned}
$$


The second equality has been obtained by a simple change of variables. The second integral is convergent for $x \in(0,1)$. The first defines the function

$$
\mathcal{G}_{n, m}(\mu ; x)=\iint d \mu(r) d \mu(s) \frac{\left|p_{n}(\mu ; r) p_{m}(\mu ; s)\right|}{|r-s|^{x}},
$$

As in Sect. 5, we define

Definition 11 The correlation dimensions of $\mu, d_{n m}(\mu)$, are the divergence $a b$ scissas of the integrals $\mathcal{G}_{n m}(\mu ; z)$, and the asymptotic exponents $\alpha_{n m}(\mu)$ are the divergence abscissas of the integrals $I_{n m}(\mu ; z)$.

As a matter of fact, they are here related in a simple way:

Proposition 6 For all $n, \alpha_{n n}(\mu)=d_{n n}(\mu) \geq D_{2}(\mu)$. For $n \neq m, d_{n m}(\mu) \geq$ $D_{2}(\mu)$ and $\alpha_{n m}(\mu)=\min \left\{d_{n m}(\mu), 1\right\}$.

Proof. Part of the proof is similar to that of Prop. 1 and is therefore omitted. Write Holder's inequality for the integral (47):

$$
\left|\mathcal{G}_{n, m}(\mu ; x)\right| \leq\left(\int d \mu(r)\left|p_{n}(\mu ; r)\right|^{q} \int d \mu(s)\left|p_{m}(\mu ; s)\right|^{q}\right)^{1 / q}\left(\iint \frac{d \mu(r) d \mu(s)}{|r-s|^{p x}}\right)^{1 / p},
$$

with $p^{-1}+q^{-1}=1$. For any $q>1$ the simple integrals in the above are convergent, since the measure $\mu$ possesses an infinite sequence of orthogonal polynomials. The double integral, on its part, is convergent for $p x<D_{2}(\mu)$, that is, $x<(1-$ $1 / q) D_{2}(\mu)$. The divergence abscissa of $\mathcal{G}_{n, m}(\mu ; z)$ is therefore larger than $D_{2}(\mu)-\varepsilon$ for any $\varepsilon>0$.

Next, if $n=m, d_{n n}$ is the correlation dimension of the measure $d \nu(r)=$ $\left|p_{n}(\mu ; r)\right| d \mu(r)$. Therefore, because of Frostman theorem, it is smaller than, or equal to one. It then coincides with $\alpha_{n n}$. This fact cannot be ascertained in the case $n \neq m$.

Remark 10 Notice that in the previous proof the existence of moments of arbitrary order is crucial to obtain the last inequality in the thesis, even for finite $n, m$. In the proof of the parallel Prop. 1 a finite set of $n$ values did only require the existence of a finite set of moments. The reason behind this asymmetry is that the functions $p_{n}(\mu ; s) p_{m}(\mu ; s)$ are not the orthogonal polynomials of the correlation measure $\Omega$ of eq. (14), unless, of course, $n=m=0$.

Remark 11 The inequality $d_{n n}(\mu) \geq D_{2}(\mu)$ can be strict, as the following example shows: let $d \mu(s)=|s|^{-\theta} d s$ on $[-1,1]$, with $\theta \in(1 / 2,1)$. Then, explicit calculation gives $D_{2}(\mu)=2(1-\theta)<1$ and $d_{11}(\mu)=1$.

We can now prove the general decay theorem:.

Theorem 10 Let $\alpha_{n m}(\mu)$ be the asymptotic exponents in Def. 11. For all $x$ such that $x<\alpha_{n m}(\mu)$, when $t \rightarrow \infty$, the asymptotic estimate $A_{n m}(\mu ; t)=o\left(t^{-x}\right)$ holds.

Proof. Proceed as in the proof of Thm. 6, using the results of Prop. 6. 


\section{Analyticity of the Mellin Transform and decay theorems}

We now study the properties of the Mellin transform of quadratic amplitudes, its inversion, and the consequences on decay estimates.

Proposition 7 The integral representation (45) defines an analytic function in the domain $0<\Re(z)<\alpha_{n m}(\mu)$. Moreover, in the same domain, the function $t \rightarrow$ $t^{z-1} A_{n m}(\mu ; t)$ belongs to $L^{1}([0, \infty], d t)$. Finally, the Mellin transform of $A_{n m}(\mu ; t)$, $M_{n m}(\mu ; z)$ can be written

$$
M_{n m}(\mu ; z)=G_{n m}(\mu ; z) H(z),
$$

where $H(z)$ has been defined in eq. (27) and where $G_{n m}(\mu ; z)$ is defined by the integral representation

$$
G_{n m}(\mu ; z):=\iint d \mu(r) d \mu(s) \frac{p_{n}(\mu ; r) p_{m}(\mu ; s)}{|r-s|^{z}} .
$$

Proof. All statements are consequence of Prop. 6 and of Fubini theorem. The proof is similar to that of Prop. 2 and is therefore omitted.

A consequence of Proposition (7) is

Lemma 5 The divergence abscissa of the Mellin transform $M_{00}(\mu ; z)$ coincides with $D_{2}(\mu)$.

Proof. This follows easily from the factorization (48), valid in the domain $0<$ $\Re e(z)<D_{2}(\mu)$. Since $G_{00}(\mu ; z)$ diverges at $D_{2}(\mu)$, and $D_{2}(\mu)$ is smaller than, or equal to one, so necessarily does $M_{00}(\mu ; z)$.

We can now easily generalize the results of sections 7 , and 8 to the case of quadratic amplitudes. The analogues of Prop. 3 and of Lemmas 1 to 3 follow from an almost verbatim translation. We so arrive at:

Theorem 11 For any $0<x<\min \left\{\frac{1}{2}, \alpha_{n m}(\mu)\right\}$ the quadratic amplitudes can be written as $A_{n m}(\mu ; t)=t^{-x} s_{n m}(x ; t)$, where $s_{n m}(x ; t)$ belongs to $\mathcal{C}_{0}\left(\mathbb{R}_{+}, t\right)$. Moreover, $t^{\bar{x}} A_{n m}(\mu ; t) \in L^{2}\left(\mathbb{R}_{+}, \frac{d t}{t}\right)$ for any $\bar{x}, 0<\bar{x}<\alpha_{n m}(\mu)$.

Proof: is analogous to that of Thms. 7,8 .

Theorem 11 is therefore straightforward generalization of [5], and shares the same weakness, when $\alpha_{n m}(\mu)>1 / 2$.

\section{Asymptotic Decay of Physical Amplitudes}

When $n=m$, the quantities $A_{n m}(\mu ; t)$ take on the physical meaning of quantum amplitudes, that is, of occupation probabilities (see paper II). In this case, positivity properties apply and permit to obtain the decay in strong form. 
Theorem 12 The Cesaro averages $A_{n n}(t)$ of the physical probability amplitudes, have the asymptotic behaviour $A_{n n}(t)=o\left(t^{-x}\right)$, as $t \rightarrow+\infty$, for any $0<x<$ $\alpha_{n n}(\mu)$, where $\alpha_{n n}(\mu)$ is the divergence abscissa of the electrostatic energy of $\left|p_{n}(\mu, r)\right| \mu$, defined in Def. 11 .

Proof. It is apparent from eq. (5) that $A_{n n}(t)$ is the Cesaro average of a positive function. Let us choose arbitrarily a value $t=t_{*}$, and let $\xi=A_{n n}\left(t_{*}\right)>0$. Let us now study the behaviour of $A_{n n}(t)$ in the interval between $t_{*}$ and infinity. Clearly, the fastest decay of $A_{n n}$ at zero at zero is obtained when the integrand of the Cesaro average is null for $t^{\prime}>t_{*}$. Let therefore $\tilde{A}_{n n}(t)$ be the function defined by $\tilde{A}_{n n}\left(t_{*}\right)=A_{n n}\left(t_{*}\right), \frac{d}{d t} \tilde{A}_{n n}(t)=-\frac{1}{t} \tilde{A}_{n n}(t)$, for $t^{\prime}>t_{*}$. Obviously, $\tilde{A}_{n n}(t)=\frac{\xi t_{*}}{t}$, and $A_{n n}(t) \geq \tilde{A}_{n n}(t)$, for $t \in\left[t_{*}, \infty\right]$. This fact, and Propositions 6, 7, imply that for $0<x<\bar{d}_{n n}(\mu)$ there exists a finite constant $B$ such that

$$
B=\int_{0}^{\infty} d t t^{x-1} A_{n n}(t)>\int_{t_{*}}^{\infty} d t t^{x-1} \tilde{A}_{n n}(t)=\frac{\xi t_{*}^{x}}{1-x} .
$$

Recall now that $\xi=A_{n n}\left(t_{*}\right)$ : then, the thesis follows.

In the same way, positivity properties alone allow to overcome the limitations of the technique of Sect. 11.

Lemma 6 Suppose that $f$ is the Cesaro average of a positive function g:

$$
f(t)=\frac{1}{t} \int_{0}^{t} d s g(s), g(s)>0 .
$$

Suppose also that $f(t)=t^{-x} s(t)$, where $s$ belongs to $L^{2}\left((0, \infty), t^{-1} d t\right)$, and $0<$ $x<1$. Then,

$$
f(t)=t^{-x} o(t) \text { as } t \rightarrow \infty .
$$

Proof. We can proceed as in the proof of Thm. 12: Let us choose quite arbitrarily a value $t=t_{*}$, and let $\xi=f\left(t_{*}\right)>0$, to obtain $f(t) \geq \tilde{f}(t)=\frac{\xi t_{*}}{t}$, $t \in\left[t_{*}, \infty\right]$. Therefore,

$$
\int_{t_{*}}^{\infty} \frac{d t}{t} s^{2}(t) \geq \int_{t_{*}}^{\infty} d t t^{2 x-1}(\tilde{f}(t))^{2}=\left(t_{*} \xi\right)^{2} \int_{t_{*}}^{\infty} d t t^{2 x-3}=\frac{t_{*}^{2 x} \xi^{2}}{2-2 x} .
$$

Finally, employing the second hypothesis of the Lemma we see that given any $M>0$, for all $t_{*}$ larger than a certain $T$ we have

$$
\int_{t_{*}}^{\infty} \frac{d t}{t} s^{2}(t) \leq M
$$

which implies that, under the same conditions, $\xi \leq \sqrt{M(2-2 x)} t_{*}^{-x}$ Recalling now that $\xi=f\left(t_{*}\right)$ the thesis follows. 
Remark 12 This lemma permits us to prove in a different way Thm. 12: Alternative proof of Thm. 12. Since physical amplitudes are obtained setting $n=m$ in eq. (44), the integrand is visibly a positive function. Theorem 11 and Lemma 6 then apply.

Remark 13 A similar trick can be applied to the averaged F-B. functions $\overline{\mathcal{J}}_{n}(\mu ; t)$, relying on theorem 8 and on their boundedness. Since this latter is - for this aimweaker than positivity, it leads to the result $\overline{\mathcal{J}}_{n}(\mu ; t)=t^{-\frac{2}{3} x} o(t)$ as $t \rightarrow \infty$, for any $x<\alpha_{n}(\mu)$. Clearly, this estimate is of lesser significance than those obtained in Section 9.

\section{Asymptotic Decay of Quadratic Amplitudes}

In the previous section we have shown that inversion theory can be augmented with positivity properties to obtain the strong asymptotic behaviour of the Cesaro averages of physical amplitudes, that is, the diagonal case $n=m$. As to the nondiagonal amplitudes, inversion theory leads to a weaker result (Thm. 11) than the direct Thm. 10. This can be obviated by a generalization of the technique of Sect. 9 , to treat conveniently also the non-diagonal entries $A_{n m}(\mu ; t), n \neq m$.

Proposition 8 For $0<\Re(z)<\alpha_{n m}(\mu)$ the Mellin transforms of $\mathcal{J}_{n}(\mu ; t) \mathcal{J}_{m}^{*}(\mu ; t)$ has a convergent integral representation, and it can be represented as

$$
\int_{0}^{\infty} t^{z-1} \mathcal{J}_{n}(\mu ; t) \mathcal{J}_{m}^{*}(\mu ; t)=\Gamma(z) e^{i \frac{\pi}{2} z} G_{n m}(\mu ; z) .
$$

Proposition 9 For $\Re(z)<\min \left\{d_{n, m}(\mu), 2\right\}$, the Mellin transform $M_{n m}(\mu ; z)$ exists as a convergent improper Riemann integral, and it can be represented as in Proposition 7, eqs. (27), (48),(49).

Proof. It is analogous to that of Prop. 4.

Remark 14 Finally, we can obtain:

Alternative proof of Thm. 10. Apply Thm. 9 with $g(t)=\mathcal{J}_{n}(\mu ; t) \mathcal{J}_{m}^{*}(\mu ; t)$.

We have therefore completed the study of the large time asymptotics of the Cesaro averages of F-B. functions, and of quadratic amplitudes.

\section{Asymptotics of the Mellin Transform in the Strip of Analyticity}

It now our goal to study the asymptotic behaviour of the Mellin transform $M_{n}(\mu ; x+$ $i y)$ itself, for large imaginary argument in the strip of analyticity. Indeed, since $H(z)$ does not depend on $\mu$, and features the asymptotic behaviour in eq. (33), we 
are left with the problem of studying $G_{n}(\mu ; x+i y)$, for $x<\alpha_{n}(\mu)$, and large $|y|$. We shall later make the hypothesis that the support of the measure $\mu$ is bounded.

The function $G_{0}(\mu ; x+i y)$ can itself be considered as a Fourier transform of a measure, as it follows from its definition in eq. (20):

$$
G_{0}(\mu ; x+i y)=\int d \mu(s) \frac{1}{|s|^{x+i y}}=\int d \nu_{x}(\mu ; \tau) e^{-i y \tau} .
$$

We shall discuss below the modifications to be made to treat the case of $n \neq 0$. The measure $\nu_{x}$ is constructed upon $\mu$, and can be defined by Riesz representation theorem via the equation

$$
\int d \nu_{x}(\mu ; \tau) f(\tau)=\int d \mu(s)|s|^{-x} f(\log (|s|))
$$

that is required to hold for any continuous function $f$ which vanishes at infinity.

Theorem 13 Let $x<\alpha_{0}(\mu)$. The Cesaro average in the variable $y$ up to the limit $t$ of the function $G_{0}(\mu ; x+i y)$ decays asymptotically as $o\left(t^{-a}\right)$, for any $a<$ $\min \left\{d_{0}\left(\nu_{x}\right), 1\right\}$. Here, $d_{0}\left(\nu_{x}\right)$ is the divergence abscissa of the integral $\mathcal{G}_{\nu}(w)$,

$$
\mathcal{G}_{\nu}(w)=\int d \mu(r)|r|^{-x}|\log | r||^{-w}
$$

The quadratic amplitude $\left|G_{0}(\mu ; x+i y)\right|^{2}$ decays asymptotically, in Cesaro average in the variable $y$ up to the limit $t$, as $o\left(t^{-a}\right)$, for any $a<D_{2}\left(\nu_{x}\right)$, where $D_{2}\left(\nu_{x}\right)$ is the divergence abscissa of the double integral $\mathcal{E}\left(\nu_{x} ; w\right)$ :

$$
\mathcal{E}\left(\nu_{x} ; w\right)=\iint d \mu(r) d \mu(s) \frac{1}{\left.|r|^{x}|s|^{x}|\log | r|-\log | s\right|^{w}} .
$$

Proof: Notice that, actually, $d_{0}\left(\nu_{x}\right)$ is the local dimension of $\nu_{x}$ at $\tau=0$. In fact, this latter is defined by the divergence abscissa of the integral

$$
\int d \nu_{x}(\tau) \frac{1}{|\tau|^{w}}
$$

We now show that this latter integral is equal to $\mathcal{G}_{\nu}(w)$. In fact, by the Beppo Levi theorem,

$$
\int d \nu_{x}(\tau) \frac{1}{|\tau|^{w}}=\lim _{\varepsilon \rightarrow 0} \int d \nu_{x}(\tau) \chi_{\varepsilon}(\tau) \frac{1}{|\tau|^{w}}
$$

where $\chi_{\varepsilon}(\tau)$ is a continuous function, equal to one everywhere except in a neighborhood of zero of size $\varepsilon$, and null in a neighborhood of zero of size $\varepsilon / 2$. By the representation (52), the last limit is equal to

$$
\lim _{\varepsilon \rightarrow 0} \int d \mu(s)|s|^{-x} \frac{1}{|\log | s||^{w}} \chi_{\varepsilon}(\log |s|)=\int d \mu(s)|s|^{-x} \frac{1}{|\log | s||^{w}} .
$$


The last equality in eq. (57) follows again via Beppo Levi theorem. A similar argument shows that the integral $\mathcal{E}\left(\nu_{x} ; w\right)$ is equal to the generalized electrostatic energy of the measure $\nu_{x}$ :

$$
\iint \frac{d \nu_{x}(r) d \nu_{x}(s)}{|r-s|^{w}}=\iint d \mu(r) d \mu(s) \frac{1}{|r|^{x}|s|^{x}|\log | r|-\log | s||^{w}}=\mathcal{E}\left(\nu_{x} ; w\right) .
$$

To conclude the proof, apply Thms. 6 and 10 .

We now study the generalised dimension $D_{2}\left(\nu_{x}\right)$ as a function of $x$. In the course of this work we shall need a couple of technical lemmas, the first of which is of some interest of its own.

Proposition 10 Let $\alpha, \beta$ be positive measures. The electrostatic correlation dimension of $\alpha+\beta$ is the minimum of the electrostatic correlation dimensions of $\alpha$ and $\beta$ :

$$
D_{2}(\alpha+\beta)=\min \left\{D_{2}(\alpha), D_{2}(\beta)\right\}
$$

Proof. Define the following symmetric bilinear quadratic form over the vector space of real measures:

$$
(\alpha, \beta):=\iint d \alpha(r) d \beta(s) \frac{1}{|r-s|^{x}} .
$$

The electrostatic energy of $\alpha+\beta$ is the divergence abscissa of $(\alpha+\beta, \alpha+\beta)$. Clearly,

$$
(\alpha+\beta, \alpha+\beta)=(\alpha, \alpha)+(\beta, \beta)+2(\alpha, \beta)
$$

Since $\alpha$ and $\beta$ are positive measures $(\alpha, \beta) \geq 0$, and so

$$
(\alpha+\beta, \alpha+\beta) \geq(\alpha, \alpha)+(\beta, \beta)
$$

This relation implies that $D_{2}(\alpha+\beta)$ is smaller than the minimum of $D_{2}(\alpha), D_{2}(\beta)$.

Now the reverse inequality [28]. Observe that the quadratic form is positive: in fact, let $\sigma$ be any signed real measure, and let $\hat{\sigma}$ be its Fourier transform:

$$
\hat{\sigma}(t):=\int d \sigma(s) e^{-i t s}
$$

Clearly, $|\hat{\sigma}(t)|^{2}$ is a positive quantity, and so is its Cesaro average, $\mathcal{C}\left(|\hat{\sigma}(t)|^{2}\right)$. Let $g(z)$ be the Mellin transform of this latter function. For $x$ real, $0<x<1, g(x)$ is also positive. The calculations performed in the previous sections can be carried over also for a signed measure, showing that

$$
g(x)=\mathcal{E}(\sigma ; x) \Gamma(x) \cos \left(\frac{\pi}{2} x\right) .
$$

It then follows that $\mathcal{E}(\sigma ; x)$ is also positive, for $x \in(0,1)$, and for any signed real measure $\sigma$. Then $(\sigma, \sigma)=\mathcal{E}(\sigma ; x)$ is also positive. 
Positivity of the quadratic form $(\cdot, \cdot)$ is required to apply Schwartz inequality to eq. (60), to get

$$
(\alpha+\beta, \alpha+\beta) \leq(\alpha, \alpha)+(\beta, \beta)+2(\alpha, \alpha)^{1 / 2}(\beta, \beta)^{1 / 2}
$$

from which $D_{2}(\alpha+\beta) \geq \min \left\{D_{2}(\alpha), D_{2}(\beta)\right\}$.

Lemma 7 Let $\mu$ be a positive measure. Let $\mu_{e}(s):=\mu(s)+\mu(-s), s \in \mathbb{R}_{+}$be its symmetrization. Then, $D_{2}\left(\mu_{e}\right)=D_{2}(\mu)$.

Proof: First let $\mu_{+}$and $\mu_{-}$be the restriction of $\mu$ to $\mathbb{R}_{+}$and its complementary, respectively, so that $\mu=\mu_{+}+\mu_{-}$. Prop. 10 implies that $D_{2}(\mu)=\min \left\{D_{2}\left(\mu_{+}\right), D_{2}\left(\mu_{-}\right)\right\}$. Consider now the measure $\overline{\mu_{-}}$defined via $\overline{\mu_{-}}(s)=\mu_{-}(-s)$. This measure is supported on the positive axis. Clearly, $D_{2}\left(\overline{\mu_{-}}\right)=D_{2}\left(\mu_{-}\right)$. Now, on the positive axis, $\mu_{e}=\mu_{+}+\overline{\mu_{-}}$. Since $\mu_{e}$ is symmetric, $D_{2}\left(\mu_{e}\right)=D_{2}\left(\mu_{+}+\overline{\mu_{-}}\right)=$ $\min \left\{D_{2}\left(\mu_{+}\right), D_{2}\left(\mu_{-}\right)\right\}=D_{2}(\mu)$.

We are now ready for

Theorem 14 Let $\mu$ have bounded support. Let $D_{2}\left(\nu_{x}\right)$ be the electrostatic correlation dimension of the measure $\nu_{x}$ defined via eq. (52), and let $0 \leq x \leq x^{\prime}<d_{0}(\mu)$. Then,

$$
D_{2}\left(\nu_{x^{\prime}}\right) \leq D_{2}\left(\nu_{x}\right)
$$

Moreover,

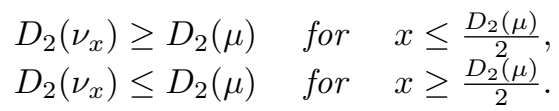

Proof. The electrostatic energy $\mathcal{E}\left(\nu_{x} ; w\right)$ of the measure $\nu_{x}$ is defined by the double integral (54). The electrostatic correlation dimension is the divergence abscissa of $\mathcal{E}\left(\nu_{x} ; w\right)$, that can be computed restricting $w$ to the real axis: $w \in \mathbb{R}$. Since we can assume by a proper rescaling that $|s|,|r|<1$, the inequality $x \leq x^{\prime}$ implies also $|r|^{x} \geq|r|^{x^{\prime}}$, and similarly $|s|^{x} \geq|s|^{x^{\prime}}$, so that

$$
\mathcal{E}\left(\nu_{x} ; w\right) \leq \mathcal{E}\left(\nu_{x^{\prime}} ; w\right) \text {. }
$$

It then follows that the divergence abscissa of the second function is smaller than that of the first: this is the inequality (62).

More work is required to get the inequalities (63). Let us therefore derive a series of estimates. Firstly,

$$
\frac{\| r|-| s||}{\max \{|r|,|s|\}} \leq|\log | r|-\log | s|| \leq \frac{\| r|-| s||}{\min \{|r|,|s|\}} .
$$

These inequalities enforce the following bounds:

$$
\mathcal{E}\left(\nu_{x} ; w\right) \leq \mathcal{U}(x ; w):=\iint d \mu(r) d \mu(s) \frac{\max \{|r|,|s|\}^{w}}{\left.|r|^{x}|s|^{x}|| r|-| s\right|^{w}}
$$


and

$$
\mathcal{E}\left(\nu_{x} ; w\right) \geq \mathcal{L}(x ; w):=\iint d \mu(r) d \mu(s) \frac{\min \{|r|,|s|\}^{w}}{\left.|r|^{x}|s|^{x}|| r|-| s\right|^{w}}
$$

Moreover, let us compute

$$
\mathcal{U}(x ; w)-\mathcal{L}(x ; w)=\iint d \mu(r) d \mu(s) \frac{\max \{|r|,|s|\}^{w}-\min \{|r|,|s|\}^{w}}{\left.|r|^{x}|s|^{x}|| r|-| s\right|^{w}} .
$$

Because of symmetry, we get

$$
\mathcal{U}(x ; w)-\mathcal{L}(x ; w) \leq 2 \iint_{|s| \leq|r|} d \mu(r) d \mu(s) \frac{|r|^{w}-|s|^{w}}{\left.|r|^{x}|s|^{x}|| r|-| s\right|^{w}} .
$$

It can be easily verified that, when $|s| \leq|r|$, and $0<w \leq 1$, one has

$$
0 \leq \frac{|r|^{w}-|s|^{w}}{\left.|| r|-| s\right|^{w}} \leq 1
$$

Then,

$$
\mathcal{U}(x ; w)-\mathcal{L}(x ; w) \leq 2 \iint_{|s| \leq|r|} d \mu(r) d \mu(s) \frac{1}{|r|^{x}|s|^{x}} \leq 2\left(\int d \mu(r) \frac{1}{|r|^{x}}\right)^{2} .
$$

Since $x<d_{0}$, the last term at r.h.s. is finite, and so is the difference $\mathcal{U}(x ; w)-$ $\mathcal{L}(x ; w)$ for any value of $0<w \leq 1$. Therefore, the integrals $\mathcal{U}(x ; w), \mathcal{L}(x ; w)$ and $\mathcal{E}\left(\nu_{x} ; w\right)$ are all either convergent, or divergent, when $0<w \leq 1$.

Let us now work on $\mathcal{U}(x ; w)$. Let $M:=\max \{|r|,|s|\}$ and $m:=\min \{|r|,|s|\}$. Clearly,

$$
\mathcal{U}(x ; w)=\iint d \mu(r) d \mu(s) \frac{M^{w-x}}{\left.m^{x}|| r|-| s\right|^{w}} \geq \iint d \mu(r) d \mu(s) \frac{M^{w-2 x}}{\|r|-| s\|^{w}} .
$$

If now $w<2 x$ we can bound $M^{w-2 x} \geq 1$, and

$$
\mathcal{U}(x ; w) \geq \iint d \mu(r) d \mu(s) \frac{1}{\|r|-| s\|^{w}}:=\mathcal{E}\left(\mu_{e} ; w\right),
$$

where $\mathcal{E}\left(\mu_{e} ; w\right)$ is the electrostatic energy of the measure $\mu_{e}$. Therefore, if $D_{2}\left(\mu_{e}\right)=$ $D_{2}(\mu)$ is less than $2 x, \mathcal{U}(x ; w)$ is also divergent at $w=D_{2}(\mu)$, or possibly before: the same goes for $\mathcal{E}\left(\nu_{x} ; w\right)$, and proves the second estimate in (63).

Similar arguments can be used for $\mathcal{L}(x ; w)$ :

$$
\mathcal{L}(x ; w)=\iint d \mu(r) d \mu(s) \frac{m^{w-x}}{\left.M^{x}|| r|-| s\right|^{w}} \leq \iint d \mu(r) d \mu(s) \frac{m^{w-2 x}}{\|\left. r|-| s\right|^{w}} .
$$

Let us now assume that $w>2 x$, so that $m^{w-2 x} \leq 1$ :

$$
\mathcal{L}(x ; w) \leq \iint d \mu(r) d \mu(s) \frac{1}{\|r|-| s\|^{w}}:=\mathcal{E}\left(\mu_{e} ; w\right) .
$$


Therefore, if $D_{2}\left(\mu_{e}\right)=D_{2}(\mu)<2 x, \mathcal{L}(x ; w)$ is convergent for all $w<D_{2}(\mu)$, and so is $\mathcal{E}\left(\nu_{x} ; w\right)$. The divergence abscissa $D_{2}\left(\nu_{x}\right)$ is therefore larger than $D_{2}(\mu)$ : this is the first estimate in (63).

Remark 15 A consequence of this theorem is that $D_{2}\left(\nu_{D_{2}(\mu) / 2}\right)=D_{2}(\mu)$. If $D_{2}\left(\nu_{x}\right)$ does not actually depend on $x$, then it must be equal to $D_{2}(\mu)$. This can be proven to be the case of a class of I.F.S. measures that we shall examine in a companion paper.

Remark 16 The previous analysis can be extended to treat all functions $G_{n}(\mu ; x+$ $i y$ ), under the simplifying assumption of boundedness of the support of $\mu$. First, define $\nu_{x,+}$, and $\nu_{x,-}$ via a variant of eq. (52),

$$
\int d \nu_{x, \pm}(\tau) f(\tau)=\int d \mu_{ \pm}(s)|s|^{-x} f( \pm \log (|s|)) .
$$

Then,

$$
G_{n}(\mu ; x+i y)=\sum_{ \pm} \int d \nu_{x, \pm}(\tau) e^{-i y \tau} p_{n}\left(\mu ; \pm e^{\tau}\right) .
$$

On the support of $\nu_{x, \pm}$, the functions $p_{n}\left(\mu ; \pm e^{\tau}\right)$ are bounded by the constants $C_{n}=\left\|p_{n}\right\|_{L_{\infty}(d \mu)}$, and the analysis of this section carries over.

\section{$15 L^{1}$ property of the Mellin transform in the strip of analyticity}

Apart from being interesting in itself, the study of the previous section is instrumental in establishing larger domains of validity of the $L^{1}$ property of $M_{n}(\mu ; x+i y)$ than those obtained in Lemma 2. In fact, the content of Thm. 13 can be used to show the following:

Lemma 8 For any $x<d_{0}(\mu)$ there exists $\eta(x)>0$ such that the Cesaro average of $\left|G_{n}(\mu ; x+i y)\right|$ verifies

$$
\frac{1}{2 t} \int_{-t}^{t} d y\left|G_{n}(\mu ; x+i y)\right|=o\left(t^{-\eta(x) / 2+\varepsilon}\right),
$$

for any $\varepsilon>0$. The quantity $\eta(x)$ is the electrostatic correlation dimension of $D_{2}\left(\nu_{x}\right)$ of the measure $\nu_{x}$ defined in eq. (52).

Proof. Observe that we can write

$$
\int_{-t}^{t} d y\left|G_{n}(\mu ; x+i y)\right| \leq\left(\int_{-t}^{t} d y\right)^{\frac{1}{2}}\left(\int_{-t}^{t} d y\left|G_{n}(\mu ; x+i y)\right|^{2}\right)^{\frac{1}{2}},
$$


which allows us to bound the asymptotic behaviour of the integral of the modulus of $G$ by that of its square modulus. Then, apply the results of Thm. 13:

$$
\int_{-t}^{t} d y\left|G_{n}(\mu ; x+i y)\right|^{2}=o\left(t^{1-D_{2}\left(\nu_{x}\right)+\varepsilon}\right),
$$

for any $\varepsilon>0$. The result of the Lemma then follows.

Our previous results on the $L^{1}$ property of $M_{n}(\mu ; x+i y)$ have been based on the boundedness of $G_{n}(\mu ; x+i y)$. The new result permits us to conclude that:

Theorem 15 Let $\eta(x)$ be as in Lemma 8. For all $x$ such that $x<\min \left(d_{0}(\mu), \frac{1+\eta(x)}{2}\right)$, $M_{n}(\mu ; x+i y)$ belongs to $L^{1}(\mathbb{R}, d y)$, and consequently $\overline{\mathcal{J}}_{n}(\mu ; t)=t^{-x} o(t)$, when $t \rightarrow \infty$.

Proof. Let us estimate the integral $\int_{-t}^{t} d y|H(x+i y)|\left|G_{n}(\mu ; x+i y)\right|$. Now, the asymptotic estimate (33) must be combined with the result of Lemma 8: the former implies that there exists $W>0$ so that, for $|y|>W,|H(x+i y)| \leq 2|y|^{-\frac{3}{2}+x}$. Splitting then the integral above, we obtain

$$
\int_{-t}^{t} d y|H(x+i y)|\left|G_{n}(\mu ; x+i y)\right| \leq B+2 \int_{t>|y|>W} d y|y|^{-\frac{3}{2}+x}\left|G_{n}(\mu ; x+i y)\right|,
$$

where $B$ is a constant. The last integral can be now computed by parts, obtaining

$$
\int_{t>|y|>W} d y|y|^{-\frac{3}{2}+x}\left|G_{n}(\mu ; x+i y)\right|=B^{\prime}+o\left(t^{x-\frac{1}{2}(1+\eta(x))}\right),
$$

where $B^{\prime}$ is another constant, whence the first part of the thesis follows. The second part can be proven along the lines of Theorem 7 .

Note that the range of values of $x$ is defined only in implicit form in the above theorem. Crucial to its determination is the behavior of the correlation dimension $D_{2}\left(\nu_{x}\right)=\eta(x)$.

\section{Local Analysis at an Arbitrary Point}

The analysis of the asymptotic behaviour of the Cesaro averages $\mathcal{J}_{n}(\mu ; t)$ has brought into light the rôle of the measure in the neighbourhood of the point zero. In fact, we can place zero wherever we please, by usage of the shifted measure $d \mu(\cdot-s)$, where $s$ is the value of the shift. The orthogonal polynomials of the shifted measure are easily obtained from those of the original measure. Indeed, their Jacobi matrix, $J(s)$, is given by $J(s)=J_{\mu}+s I$, where $I$ is the identity matrix, and $J_{\mu}$ is the Jacobi matrix associated with the measure $\mu$. In addition, we have that $\mathcal{J}_{n}(\mu(\cdot-s) ; t)=e^{-i t s} \mathcal{J}_{n}(\mu ; t)$, a relation that permits to obtain all the shifted zero order F-B. functions in terms of the one with null shift. These simple observations permits us to construct a spectrum analyzer tunable to detect 
the local properties of a measure $\mu$ at all points, much in the spirit of the wavelet analysis $[22,23]$.

The techniques presented so far also permit to obtain the interesting relation between the local dimensions $d_{0}(\mu ; r)$ and the correlation dimension $D_{2}(\mu)$ stated in Thm. 1. To do this, let $\mathcal{C}(f)$ be the short-hand notation for the function $\mathcal{C}(f ; T)$. We have the following

Lemma 9 Let $f$ be a complex function, $\mathcal{C}(f)$ its Cesaro average, and let us consider the Mellin transform of its modulus, $\mathcal{M}(|\mathcal{C}(f)| ; z)$. Let $d_{|\mathcal{C}(f)|}$ be its divergence abscissa. Then, this latter is larger than, or equal to, the divergence abscissa of the M.T. of the square root of the Cesaro average of $|f|^{2}$ :

$$
d_{|\mathcal{C}(f)|} \geq d_{\left(\mathcal{C}\left(|f|^{2}\right)\right)^{1 / 2}}
$$

Proof: Apply Schwartz inequality to the integral defining the Cesaro average:

$$
\left|\int_{-T}^{T} d t f(t)\right|^{2} \leq\left(\int_{-T}^{T} d t|f(t)|^{2}\right)\left(\int_{-T}^{T} d t\right)=4 T^{2} \mathcal{C}\left(|f|^{2} ; T\right) .
$$

Hence,

$$
|\mathcal{C}(f ; T)| \leq \mathcal{C}\left(|f|^{2} ; T\right)^{\frac{1}{2}}
$$

and

$$
\int_{0}^{W} d T T^{x-1}|\mathcal{C}(f ; T)| \leq \int_{0}^{W} d T T^{x-1} \mathcal{C}\left(|f|^{2} ; T\right)^{\frac{1}{2}} .
$$

Then, if the rightmost term is convergent, as $W$ tends to infinity, so is the first. This implies the thesis.

Lemma 10 Let $g$ be a positive function, bounded in [0,1], and with positive divergence abscissa of the Mellin transform, $d_{g}$. Then, this latter is related to that of $|g|^{2}, d_{|g|^{2}}$, by the inequality

$$
d_{g} \geq \frac{1}{2} d_{|g|^{2}}
$$

Proof: Apply Schwartz inequality to the integral $\int_{1}^{T} t^{u} t^{x-1-u} g(t) d t$, to get

$$
\left|\int_{1}^{T} d t t^{x-1} g(t)\right|^{2} \leq \frac{1}{2 u+1}\left(T^{2 u+1}-1\right) \int_{1}^{T} d t t^{2 x-2 u-2}|g(t)|^{2},
$$

for any $u \neq-\frac{1}{2}$. This can also be read as

$$
|M(g, x, 1, T)|^{2} \leq \frac{1}{2 u+1}\left(T^{2 u+1}-1\right) M\left(|g|^{2}, 2 x-2 u-1,1, T\right),
$$


where $M(g, x, 1, T)$ is the Mellin transform integral of the function $g$ with upper integration limit $T$ and lower integration limit one. We now let $T$ tend to infinity. Suppose that $2 u+1<0$, and put $a=-2 u-1>0$. Then, if $|M(g, x, 1, T)|$ tends to infinity, forcefully $M\left(|g|^{2}, 2 x-2 u-1,0, T\right) \geq M\left(|g|^{2}, 2 x-2 u-1,1, T\right)$ tends to infinity, too. The first condition is implied by $x>d_{g}$. Therefore, this also implies that $2 x+a>d_{|g|^{2}}$. Since this holds for any $a>0$ we obtain the thesis.

Proposition 11 The divergence abscissa of the Mellin transform of the modulus of the Cesaro average $\overline{\mathcal{J}}_{0}(\mu ; t):=\mathcal{C}\left(\mathcal{J}_{0}(\mu) ; t\right)$ is larger than, or equal to, one-half of the divergence abscissa of the Mellin transform of the Cesaro average of $\left|\mathcal{J}_{0}(\mu ; t)\right|^{2}$.

Proof: Let $g$ be the following function:

$$
g(t):=\left(\mathcal{C}\left(\left|\mathcal{J}_{0}(\mu ; \cdot)\right|^{2} ; t\right)\right)^{1 / 2} .
$$

Lemma 10 then implies that $d_{g} \geq \frac{1}{2} d_{|g|^{2}}$, where now $|g|^{2}=\mathcal{C}\left(\left|\mathcal{J}_{0}(\mu)\right|^{2}\right)$. In addition, using Lemma 9,

$$
d_{g}=d_{\left(\mathcal{C}\left(\left|\mathcal{J}_{0}(\mu)\right|^{2}\right)\right)^{1 / 2}} \leq d_{\left|\mathcal{C}\left(\mathcal{J}_{0}(\mu)\right)\right|},
$$

and the thesis follows.

Lemma 11 When $d(\mu ; s)<1$, the divergence abscissa of the Mellin transform of the modulus of the Cesaro average of $\mathcal{J}_{0}(\mu(\cdot-s) ; t)$ coincides with $d(\mu ; s)$.

Proof: Let for simplicity of notation $s=0$. Recall that, when $d_{0}(\mu)<1$, one has $\alpha_{0}(\mu)=d_{0}(\mu)$. From the definition of $I_{0}(x)$, eq. (18), and letting $x<d_{0}(\mu)$, we get:

$$
\begin{gathered}
I_{0}(\mu ; x):=\int d \mu(r) \int_{0}^{\infty} d t\left|t^{x-1} \frac{\sin t r}{t r}\right| \geq \int_{0}^{\infty} d t t^{x-1}\left|\int d \mu(r) \frac{\sin t r}{t r}\right|= \\
\left.=\mathcal{M}\left(\left|\overline{\mathcal{J}}_{0}(\mu)\right| ; x\right)\right) \geq\left|\int_{0}^{\infty} d t t^{x-1} \int d \mu(r) \frac{\sin t r}{t r}\right|=\left|\mathcal{M}\left(\overline{\mathcal{J}}_{0}(\mu) ; x\right)\right|
\end{gathered}
$$

The divergence abscissa of $I_{0}(x), \alpha_{0}(\mu)$, is therefore smaller than, or equal to, the divergence abscissa of $\left.\mathcal{M}\left(\left|\overline{\mathcal{J}}_{0}(\mu)\right| ; x\right)\right)$. Now we prove the reverse inequality. Since $x<\alpha_{0}(\mu)$, the representation $\mathcal{M}\left(\overline{\mathcal{J}}_{0}(\mu) ; x\right)=G_{0}(\mu ; x) H(x)$ holds. When $x$ tends to $\alpha_{0}(\mu), G_{0}(\mu ; x)$ diverges, and so does $\left|\mathcal{M}\left(\overline{\mathcal{J}}_{0}(\mu) ; x\right)\right|$, and $\left.\mathcal{M}\left(\left|\overline{\mathcal{J}}_{0}(\mu)\right| ; x\right)\right)$ as well. But this means that the divergence abscissa of this last Mellin transform is smaller than, or equal to $\alpha_{0}(\mu)$.

An interesting corollary follows:

Proposition 12 When $d_{0}(\mu)<1$ :

$$
\limsup _{t \rightarrow \infty} \frac{\log C_{1}\left(\left|\overline{\mathcal{J}}_{0}(\mu)\right| ; t\right)}{\log t}=-d_{0}(\mu) .
$$


Proof. Since the modulus of $\overline{\mathcal{J}}_{0}(\mu)$ is a non-negative function, we can apply Thm. 4.

This result is nonetheless a bit involved, since it considers the Cesaro average of the modulus of a Cesaro average.

We are now ready to prove Thm. 1 of Sect. 1 , that asserts that the local dimension of $\mu$ at any point $s$ is larger than, or equal to, one-half of the electrostatic correlation dimension:

$$
d(\mu ; s) \geq \frac{1}{2} D_{2}(\mu) \quad \forall s \in \mathbb{R} .
$$

Proof of Thm. 1 in Sect. 1: The electrostatic correlation dimension of the measure $\mu$ is the divergence abscissa of the generalized electrostatic energy $\mathcal{E}(\mu ; z)$, eq. (8). It is also the divergence abscissa of the Mellin transform of the Cesaro average of $\left|\mathcal{J}_{0}(\mu ; t)\right|^{2}$, as proven in Sect. 10 above.

The local dimensions $d(\mu ; s)$ are the divergence abscissas of the singular integrals $\mathcal{G}(\mu ; s, z)$, eq. (7). It follows from Lemma 11 that, when $d(\mu ; s)<1$ these quantities coincide with the divergence abscissa of the Mellin transform of the modulus of the Cesaro average of $\mathcal{J}_{0}(\mu(\cdot-s) ; t)=e^{-i t s} \mathcal{J}_{0}(\mu ; t)$.

Then, two cases must be considered. If $d(\mu ; s) \geq \frac{1}{2}$, then forcefully $d(\mu ; s) \geq$ $\frac{1}{2} D_{2}$, since $D_{2}(\mu)$ is always smaller than, or equal to, one. On the other hand, when $d(\mu ; s)<\frac{1}{2}$ the identification of the divergence abscissas of the spectral quantities $(8,7)$ with their dynamical analogue is valid, we can apply Proposition 11, and the thesis follows.

A few comments to this result are in order. First of all, the validity for all points $s$ is to be remarked. This is to be compared with the almost certain statement:

Proposition 13 The local dimensions of $\mu$ at $\mu$-almost all points $s, d(\mu ; s)$, are larger than, or equal to, the electrostatic correlation dimension of the measure $\mu$, $D_{2}(\mu)$ :

$$
d(\mu ; s) \geq D_{2}(\mu) \mu \text {-a.e. } s \in \mathbb{R} .
$$

Proof. For $\Re(z)<D_{2}(\mu)$ the integral $\mathcal{E}(\mu ; z)$ is convergent. Therefore, it is standard to show that Fubini theorem implies that the integral $\mathcal{G}(\mu ; s, z)$, the potential at point $s$, is almost surely finite. This implies the thesis.

Remark 17 Thm. 1, and the analysis of this paper, shed light on the results of Strichartz and Last [20], that assert that if a finite Borel measure on $\mathbb{R}$ is uniformly $\alpha$ Hölder continuous (see [20] or Sect. 2 for a definition) then the Cesaro average of $\left|\mathcal{J}_{0}(\mu ; t)\right|^{2}$ (in our notation, $A_{00}(\mu ; T)$ ), decays at least as $T^{-\alpha}$, and conversely, if this is the case, then $\mu$ is uniformly at least $\alpha / 2$ continuous.

Remark 18 The inequality (9), has also been proven to hold separately for inferior and superior limit quantities within the conventional formalism [29]. Also, the weaker result of Prop. 13 is known to hold. 


\section{Conclusions}

In this paper we have developed a variety of Mellin transform techniques to analyze the asymptotic behaviour of the Cesaro averages of the F-B. functions $\mathcal{J}_{n}(\mu ; t)$ and of their products. The analysis has brought to light the rôle of suitably defined measure dimensions in defining the asymptotic decay, much in line with previous results in the literature. These results are now presented as belonging to a unified picture, that focuses on the properties of the Mellin transform. The full potential of this analysis is appreciated when applied to specific examples, like the elementary ones presented in appendix to this paper, or the case of linear iterated functions systems, to which a companion paper is devoted.

\section{Appendix}

The general theory can be exemplified on a scholastic example: the measures $d \mu(s)=s^{p} d s$ on $[0,1]$, with integer $p$. For these measures, we have $d_{0}(\mu)=1+p$. Let us first focus on the linear quantities studied in sections $5-9$.

Firstly, in the case $p=0$ one obtains the Lebesgue measure, with $\alpha_{0}(\mu)=$ $d_{0}(\mu)=1$. Letting $g(t)=\mathcal{J}_{0}(\mu ; t)$, one easily computes the Fourier transform

$$
g(t)=\frac{\sin (t)}{t}+\frac{i}{t}(\cos (t)-1)
$$

the symmetric part

$$
g_{e}(t)=\frac{\sin (t)}{t}
$$

and the Cesaro average

$$
\bar{g}(t)=\frac{S i(t)}{t} \sim \frac{\pi}{2 t} .
$$

It is apparent that $\bar{g}(t)$ behaves asymptotically as $t^{-1}$ for large $t$. Theorem 7 permits to obtain at most that the decay exponent is larger than, or equal to, one half. Theorem 8 , in weak form, asserts that $s(x, t)=t^{x} \bar{g}(t)$ belongs to $L^{2}\left((0, \infty), t^{-1} d t\right)$ for all $x$ less than one, a fact that is easily verified, and indeed, one is the superior limit of the set of $x$ values for which the $L^{2}$ property is verified. Finally, the full strong asymptotic decay can be obtained via Theorem 6 .

Let now $p=1$. Then, $d_{0}(\mu)=2$ is strictly larger than $\alpha_{0}(\mu)=1$. In this case, explicit computations provide

$$
\begin{gathered}
g(t)=\frac{e^{-i t}(i t+1)}{t^{2}}-\frac{1}{t^{2}}, \\
g_{e}(t)=\frac{1}{t^{2}}(\cos (t)-1)+\frac{1}{t} \sin (t),
\end{gathered}
$$

and

$$
\bar{g}(t)=\frac{1}{t^{2}}(1-\cos (t))
$$


The exponent of strong decay of $\bar{g}(t)$ is therefore two, and cannot be obtained via Theorem 6. Yet, as predicted by Proposition 5 , for $\Re(z)<2$, the Mellin transform of $\bar{g}(t)$ exists as an improper Riemann integral. In addition, observe that $\mathcal{M}\left(g_{e}\right)$ also exists as an improper R.I. for $\Re(z)<2$. Then, Thm. 9 asserts that $\bar{g}(t)=o\left(t^{-q^{\prime}}\right)$ for all $q^{\prime}<2$.

Finally, let $p=2$, so that $d_{0}(\mu)=3, \alpha_{0}(\mu)=1$. Here,

$$
g(t)=\frac{2 \cos (t)}{t^{2}}+\frac{\sin (t)}{t}-2 \frac{\sin (t)}{t^{3}}+i\left[\frac{2(1-\cos (t))}{t^{3}}-\frac{2 \sin (t)}{t^{2}}+\frac{\cos (t)}{t}\right],
$$

and

$$
\bar{g}(t)=\frac{\sin (t)}{t^{3}}-\frac{\cos (t)}{t^{2}} .
$$

The exponent of strong decay is still two, and it is intermediate between $\alpha_{0}(\mu)$ and $d_{0}(\mu)$.

The problem of diagonal quadratic amplitudes is simpler. Consider the case $n=m=0$ and let $g(t)=\left|\mathcal{J}_{0}(\mu ; t)\right|^{2}$. Then, the leading behaviour of $\bar{g}(t)$ is $2 \frac{S i(t)}{t}$ for $p=0, \frac{1}{3} \frac{S i(t)}{t}$ for $p=1$, and $\frac{2}{5} \frac{S i(t)}{t}$ for $p=2$. This is consistent with the fact that $D_{2}(\mu)$ is equal to one in all the three cases. 


\begin{tabular}{|c|c|c|}
\hline symbol & meaning & location \\
\hline$p_{n}(\mu ; s)$ & orthogonal polynomials of $\mu$ & eq. 1 in Sect. 1 \\
\hline $\mathcal{J}_{n}(\mu ; t)$ & ourier-Bessel functions of $\mu$ & eq. 2 in Sect. 1 \\
\hline$\overline{\mathcal{J}}_{n}(\mu ; t)$ & rage F-B. functions of $\mu$ & eq. 3 in Sect. 1 \\
\hline $\mathcal{C}(f ; t)$ & imetric Cesaro average of $f$ & eq. 3 in Sect. 1 \\
\hline & mmetric Cesaro average of $f$ & eq. 3 in Sect. 1 \\
\hline$A_{n m}(\mu ; t)$ & Iadratic amplitudes of $\mu$ & eq. 5 in Sect. 1 \\
\hline $\mathcal{M}(f ; z)$ & ellin transform of $f$ & eq. 6 in Sect. 1 \\
\hline $\mathcal{G}(\mu ; s, z)$ & ctrostatic potential of $\mu$ & Def. 3 in Sect. 1 \\
\hline $\mathcal{E}(\mu ; z)$ & neralized electrostatic energy of $\mu$ & Def. 4 in Sect. 1 \\
\hline$d(\mu ; s)$ & dimensions of $\mu$ & Def. 5 in Sect. 1 \\
\hline$D_{2}(\mu)$ & ectrostatic correlation dimension of $\mu$ & Def. 6 in Sect. 1 \\
\hline$\gamma_{ \pm}(\mu ; s)$ & per (lower) local dimensions of $\mu$ & Def. 7 in Sect. 3 \\
\hline$D_{2}^{ \pm}(\mu)$ & pper (lower) correlation dimension of $\mu$ & Def. 8 in Sect. 3 \\
\hline$M_{[0,1]}(m ; z)$ & Mellin-Stieltjes transform of $m$ on $[0,1]$ & eq. 12 in Sect. 3 \\
\hline$\Omega(\mu ; u)$ & orre & eq. 14 in Sect. 3 \\
\hline$M_{[1 \infty}$ & form of $m$ on $[1, \infty]$ & eq. 15 in Sect. 4 \\
\hline$C_{1}(m ;$ & ne sided Cesaro & eq. 16 in Sect. 4 \\
\hline $\mathcal{G}_{n}(\mu ; x)$ & of $\left|p_{n}\right| \mu$ at $z$ & eq. 20 in Sect. 5 \\
\hline$d_{n}(\mu)$ & electrostatic local dimension dimension of $\left|p_{n}\right| \mu$ at zero & eq. 21 in Sect. 5 \\
\hline$\alpha_{n}(\mu)$ & umntotic oynonont of $|n|$ & Def. 10 in Sect. 5 \\
\hline$M_{n}(\mu ; z)$ & ${ }_{2}(\mu ; t)$ & eq. 25 in Sect. 6 \\
\hline$H(z)$ & $\operatorname{sinc}$ & eq. 27 in Sect. 6 \\
\hline$G_{n}(\mu ; z)$ & ctor in $M_{n}(\mu ; z)$ & eq. 28 in Sect. 6 \\
\hline$h_{n}(x, \tau)$ & auxiliary functions & eq. 31 in Sect. 7 \\
\hline $\mathcal{C}_{0}(\mathbb{R}, \tau)$ & set of continuous functions vanishing at infinity & Prop. 3 in Sect. 7 \\
\hline $\mathcal{M}(g ; z, T)$ & truncated Mellin transform of $g$ & eq. 38 in Sect. 9 \\
\hline & mont & Lem. 4 in Sect. 9 \\
\hline$(\mu ; x)$ & $n-m$ electrostatic energy of $\mu$ & eq. 47 in Sect. 10 \\
\hline & orrelation dimensions of $\mu$ & Def. 11 in Sect. 10 \\
\hline$\alpha_{n m}(\mu)$ & $n-m$ asymptotic exponents of $\mu$ & Def. 11 in Sect. 10 \\
\hline$M_{n m}(\mu ; z)$ & Mellin transform of $A_{n m}(\mu ; t)$ & eq. 48 in Sect. 11 \\
\hline$G_{n m}(\mu ; z)$ & dynamical factor in $M_{n m}(\mu ; z)$ & eq. 49 in Sect. 11 \\
\hline & nmetrized measure & Lem. 7 in Sect. 14 \\
\hline & and to its & Lem. 7 in Sect. 14 \\
\hline & auxiliary measure & eq. 52 in Sect. 14 \\
\hline
\end{tabular}

Giorgio Mantica

Center for Non-linear and Complex Systems,

Universitá dell'Insubria

Via Valleggio 11, 22100 Como Italy

and CNISM and INFN, sez. Como

giorgio@uninsubria.it 
Sandro Vaienti

U.M.R. 6207 Centre de Physique Théorique, CNRS, Luminy, Marseille,

Universites d'Aix-Marseille I,II, Université du Sud, Toulon-Var, and Fédération de Recherche des Unités de Mathématiques de Marseille, France.

\section{References}

[1] N.I. Akhiezer, The classical moment problem and some related questions in analysys, Hafner Pub. Co., New York (1965).

[2] G. Mantica, Quantum Intermittency in Almost Periodic Systems Derived from their Spectral Properties, Physica D 103 (1997) 576-589; Wave Propagation in Almost-Periodic Structures, Physica D 109 (1997) 113-127.

[3] T. D. Sauer and J. A. Yorke, Are the dimensions of a set and its image equal under typical smooth functions? Ergod. Th. \& Dynam. Sys. 17 (1997) 941-956.

[4] B. R. Hunt and V. Yu. Kaloshin, How projections affect the dimension spectrum of fractal measures, Nonlinearity 10 (1997) 1031-1046.

[5] K.A. Makarov, Asymptotic Expansions for Fourier Transform of Singular Self-Affine Measures, J. Math. An. and App. 186 (1994) 259-286.

[6] R. S. Strichartz, Fourier asymptotics of fractal measures, J. Funct. Anal. 89 (1990) 154-187.

[7] K.S. Lau, Iterated function systems with overlaps and multifractal structure, in Trends in probability and related analysis (Taipei, 1998), 35-76, World Sci. Publishing, River Edge, NJ, 1999.

[8] K.S. Lau, Fractal measures and mean-p variations, J. Funct. Anal. 108 (1992) 427-457.

[9] K.S. Lau, Dimension of a family of singular Bernoulli convolutions, J. Funct. Anal. 116 (1993) 335-358.

[10] K.S. Lau and J. Wang, Mean quadratic variations and Fourier asymptotic of self-similar measures, Monatsh. Math. 115 (1993) 99-132.

[11] R. Strichartz, Self-similar measures and their Fourier transforms I, Indiana U. Math. J. 39 (1990) 797-817.

[12] R. Strichartz, Self-similar measures and their Fourier transforms II, Trans. Amer. Math. Soc. 336 (1993) 335-361. 
[13] D. Bessis, J.D. Fournier, G. Servizi, G. Turchetti, and S. Vaienti, Mellin transform of correlation integrals and generalized dimension of strange sets, Phys. Rev. A 36, 920-928 (1987).

[14] K. Falconer, Fractal Geometry, Wiley, Chichester (1990).

[15] E. Orlandini, M.C. Tesi, and G. Turchetti, Meromorphic structure of the Mellin transform and short-distance behavior of correlation integrals, J. Stat. Phys. 66 (1992) 515-533.

[16] R. Ketzmerick, G. Petschel, and T. Geisel, Slow decay of temporal correlations in quantum systems with Cantor spectra, Phys. Rev. Lett. 69 (1992) 695-698.

[17] J.M. Barbaroux, J.M. Combes, and R. Montcho, Remarks on the relation between quantum dynamics and fractal spectra, J. Math. Anal. Appl. 213 (1997) 698-772.

[18] C.A. Guerin and M. Holschneider, $\mathcal{L}^{2}$-Fourier asymptotic of self-similar measures, CPT preprint, Marseille, (1996).

[19] C.A. Guerin and M. Holschneider, On equivalent definitions of the correlation dimension for a probability measure, J. Stat. Phys. 86 (1997) 707-720.

[20] Y. Last, Quantum dynamics and decompositions of singular continuous spectra, J. Funct. Anal. 142 (1996), 406-445.

[21] A. Hof, On scaling in relation to singular spectra, Comm. Math. Phys. bf 184, (1997) 567 - 577.

[22] S. Vaienti and J.M. Ghez, On the wavelet analysis of multifractal sets, J. Stat. Phys. 57 (1989) 415-420.

[23] S. Vaienti, A Frostman-like theorem for the wavelet transform on fractal sets, Nonlinearity 4 (1991) 1241-1249.

[24] J. H. Zhong, J. Bellissard, and R. Mosseri, Green function analysis of energy spectra scaling properties, J. Phys.: Condens. Matter 7 (1995) 3507-3514.

[25] G. Mantica, Quantum Intermittency: Old or New Phenomenon? J. Phys. IV France 8 (1998) 253.

[26] G. Mantica, Fourier-Bessel functions of singular continuous measures and their many asymptotics, Elect. Transactions in numerical analysis, ETNA to appear (2006).

[27] Further properties of the local dimension defined in this fashion can be found in H. Schulz-Baldez and J. Bellissard, Anomalous transport: a mathematical framework, Rev. Math. Phys. 10 (1998) 1-46. 
[28] Our original proof of this inequality went a long way through Thm. 5. We thank J. Bellissard for suggesting to cut it via Schwartz inequality, and R. Strickartz for sketching to us the positivity of the quadratic form required for its application.

[29] J.M. Barbaroux, F. Germinet, S. Tcheremchantsev, Generalized fractal dimensions: equivalence and basic properties, J. Math. Pures Appl. (9) 80 (2001) 977-1012. 